\title{
CHANGES IN SOCIAL BEHAVIOR OF SQUIRREL MONKEYS AFTER TEMPORAL \\ LOBECTOMY AND AN INTERSPECIES COMPARISON OF SOCIAL DOMINANCE
}

By

RODNEY J. PLOTNIK

\begin{abstract}
A DISSERTATION PRESENTED TO THE GRADUATE COUNCIL OF THE UNIVERSITY OF FLORIDA

IN PARTIAL FULFILLMENT OF THE REQUIREMENTS FOR THE DEGREE OF DOCTOR OF PHILOSOPHY
\end{abstract}

UNIVERSITY OF FLORIDA

August, 1966 


\section{ACKNOWLEDGMENTS}

The author wishes to thank his chairman, Dr. Frederick A. King, for supervising this research. Appreciation is also expressed to Professors B. N. Bunne11, D. C. Goodman, R. L. King, and H. S. Pennypacker for their assistance as members of the doctoral committee. 
ACKNOWLEDGMENTS . . . . . . . . . . . . . . ii

IIST OF TABLES . . . . . . . . . . . . . . . . V v

LIST OF FIGURES . . . . . . . . . . . . . . . . . . . vi

INTRODUCTION . . . . . . . . . . . . . . . . . 1

MEASUREMENT AND MANIPULATION OF SD IN PRIMATES . . . . . 3

THE ROLE OF TEMPORAL LOBE STRUCTURES IN AFFECTIVE

AND SOCIAL BEHAVIOR .................. 9

Problem ....................... 19

EXPERIMENT I: SD HIERARCHY IN THE SOUTH AMERICAN

SQUIRREL MONKEY AND COMPARISON WITH REPORTS ON THE

RHESUS MONKEY ... . . . . . . . . . . . . . . 22

Part 1: Positive Reinforcement .......... . 22

Part 2: Negative Reinforcement . . . . . . . 27

Part 3: Retesting with Positive Reinforcement... . 29

Part 4: SD Hierarchy after Two-week Separation . . 30

Part 5: Reaction to a Fearful Stimulus and the

Experimenter ........... 30

EXPERIMENT II: THE EFFECT OF ANTERIOR TEMPORAL

LOBECTOMY ON THE SD BEHAVIOR OF THE SQUIRREL MONKEY . . . 37

Part 1: Changes Following White's Operation..... 45

Part 2: Changes Following Green's Operation . . . . 52

Part 3: Changes Following Red's Operation..... 58

Part 4: Changes Following Blue's Operation . . . . 64

Part 5: Comparison of Results from White, Green,

Red and Blue's Operations... . . . . . 69

Part 6: Discussion .............. 75 
EXPERIMENT III: SD HIERARCHY IN THE CEBUS

MONKEY AND COMPARISON OF SQUIRREL AND CEBUS

MONKEY EINDINGS. . . . . . . . . . . . . . . 92

Part 1: SD Hierarchy in the Cebus Monkey . . . . . . 92

Part 2: Comparative Data . . . . . . . . . 96

SUMMARY ........................ 102

CONCLUSTONS . . . . . . . . . . . . . 105

BIBLIOGRAPHY .................. 107

BIOGRAPHICAL SKETCH .................... 114 


\section{LIST OF TABLES}

Table

Page

1. The testing sequence after the dominant monkey was operated . . . . . . . . . . . . . . 38

2. The number of spontaneous crossings into the teddy bear's compartment during the five-minute exploratory period--a test for visual agnosia . . . . . 83 


\section{LIST OF FIGURES}

Figure

1. Squirrel monkey's SD hierarchy observed under four different testing conditions . . . . . . . . . 31

2. Squirrel monkey's SD hierarchy retested after completion of the negative reinforcement sequence . . . . 32

3. Squirrel monkey's SD hierarchy after two-week separation and retesting. . . . . . . . . 33

4. Reconstruction of White's lesion ............ 41

5. Reconstruction of Green's lesion . . . . . . . . . 42

6. Reconstruction of Red's lesion . . . . . . . . . 43

7. Reconstruction of Blue's lesion... . . . . . . . . 44

8. Aggressive and submissive scores for normal and operated monkeys tested in the shuttle box under positive reinforcement . . . . . . . . . . . . 84

9. Order of crossing tunnel and food pellets received in the shuttle box under positive reinforcement for normal and operated monkeys .. . . . . . . . . . 85

10. Food pellets obtained in the shuttle box and food pellets obtained or competed for in the home cage . . . 86

11. Aggressive and submissive scores for normal and operated monkeys tested in the home cage under positive reinforcement . . . . . . . . . . . 87

12. Aggressive and submissive scores for normal and operated monkeys tested in the shuttle box under negative reinforcement . . . . . . . . . . . 88

13. Order of crossing tunnel in the shuttle box under negative reinforcement for normal and operated monkeys . . . . . . . . . . . . . . . . .

14. Aggressive and submissive scores for normal and operated monkeys tested in the home cage with no experimental manipulation .............. 90

15. Total aggressive and submissive scores, across all testing conditions, for normal and operated monkeys . . 91 
16. Comparison of the SD hierarchies in the squirrel and cebus monkeys tested with positive and negative reinforcement . . . . . . . . .

17. Comparison of the SD hierarchies in the squirrel and cebus monkeys across all testing conditions . . . 98 


\section{INTRODUCTION}

A critical and difficult problem in the study of social behavior is measurement. In man, the study of social behavior is facilitated by verbal communication and is simultaneously confounded by the complexity of human behavior. At the infrahuman level, vocal communication is minimal and thus, evaluation of the complexity of social structure is made difficult both in terms of measurement and interpretation. In addition, the measurement and interpretation of infrahuman social behavior is often criticized as being anthropomorphic. Fortunately, there are multiple field studies on many infrahuman social organizations. These studies have defined and classified the naturally occurring behavioral responses which are basic to any laboratory study of social behavior.

Information from field studies can provide a referent for laboratory research on social behavior. Since the experimental method permits greater manipulation and control of the environment than does field methodology, laboratory research can make unique contributions to the study of social behavior. The first half of this investigation was concerned with the problems of measuring social behavior.

One important aspect of social behavior is social dominance (SD). SD consists of many classes of responses: emotional, per- 
ceptual, physiological and learned. Some of the behavioral components of emotional states such as rage, fear, aggression and cowering, are reported in studies of SD and are obvious in the animal's daily competition for food, territory and mates. The central question asked in the present experiments was: what specific changes would occur in SD behavior if an emotional component of an animal's behavior were altered experimentally? It is reported that destruction of the amygdala changes a wild, aggressive animal into a tame and placid one. This study was specifically concerned with the effect of bilateral amygdalectomy on SD.

The study is divided into five sections:

(1) Measurement and manipulation of SD in primates

(2) The role of temporal lobe structures in affective and social behavior

(3) Experiment I: SD hierarchy in the South American squirrel monkey and comparison with reports on the rhesus monkey

(4) Experiment II: The effect of anterior temporaI lobectomy on the SD behavior of the squirrel monkey

(5) Experiment III: SD hierarchy in the cebus monkey and comparison of squirrel and cebus monkey findings. 
MEASUREMENT AND MANIPULATION OF SD IN PRIMATES

SD is observed in primate social interactions when one monkey consistently initiates aggressive behavior or exercises priority over others in food-getting, sexual partners, and territory. SD interactions result in a social structure which is important to the formation, organization and maintenance of the group.

$\mathrm{SD}$ has been investigated in the field and laboratory. Field studies of the howler monkey (Carpenter, 1942); rhesus monkey (Altman, 1962; Southwick, 1962; Kaufman, 1966); baboon (Kummer \& Kurt, 1963); gibbon (Carpenter, 1940); gorilla (Schaller, 1963); and chimpanzee (Reynolds, 1963; Goodall, 1963) have defined SD patterns important for laboratory experimentation.

In the laboratory, SD has been studied using positive reinforcement (Miller \& Murphy, 1956; Hamilton, 1960; Plotnik et al. , 1965, 1966); negative reinforcement (Hamilton, 1960; Miller \& Banks, 1962; Plotnik et al., 1965, 1966); detailed recorded observations with and without food incentives (Ploog et al., 1963); social restriction (Mason, 1960, 1961); large outdoor runs (Bernstein, 1964, 1965, 1966); and time lapse photography (Delgado, 1962).

SD in primates has been experimentally manipulated through conditioning (Miller et al., 1955; Murphy \& Miller, 1956; Horel et al., 1963); drugs (Leary \& Slye, 1959; Leary \& Stynes, 1959); 
hormones (Mirsky, 1955; Birch \& Clark, 1950); brain lesions (Rosvold et al., 1954; Brody et al., 1952; Mirsky et al., 1957); and brain stimulation (Delgado, 1963, 1964, 1965a, 1965b).

From the laboratory and field research cited above, the following sumary statements can be made regarding the measurement and stability of SD in primates. SD can be measured by counting the reinforcements obtained in paired or group competition, and by noting the direction of aggressive interactions in a pair or group of monkeys which have or have not (task free) been manipulated. Social dominance, determined by paired competition for positive or negative incentives, is linear and stable for rhesus monkeys. A linear or straight-line hierarchy is a social arrangement in which all aggressive or dominant interaction is invariant and unidirectional. Since paired SD testing is based primarily on the number of accumulated incentives, little is reported of the social interactions between monkeys. Compared to accumulated peanuts or shocks avoided, it has been reported that social interactions such as threatening or submissive gestures, agonistic attacks, active and passive displacement, give a more accurate picture of the SD structure (Bernstein, 1964; Kaufman, 1966).

In contrast to paired testing, a more complete description of the SD structure can be obtained from group testing. A stable, linear hierarchy for adult males, less well defined for adult females and subadults, is observed in laboratory group cages, outdoor runs and free-ranging habitats for rhesus wonkeys. The social interactions observed in the laboratory show intensified aggressive 
interactions compared to free-ranging monkeys which display more passive displacement and threatening behavior than actual attack. As seen in laboratory behavior, intensified agonistic interactions are observed in free-ranging monkeys around artificial feeding stations where 98 per cent of all acts at the feeder may be aggressive compared to about 50 per cent of acts being aggressive away from the feeder (Kaufman, 1966).

Reports on the 01d World rhesus monkey consistently show a straight-line stable SD hierarchy while little is known of the New World squirrel monkey's SD structure. The first phase of the present study developed a methodology for measuring the SD structure in the squirrel and cebus monkey, and compared the squirrel monkey's SD hierarchy with reports of the rhesus monkey's hierarchy.

\section{Manipulation of SD}

Both laboratory and field studies on primates report that a SD hierarchy, once established, is generally found to be stable, although the SD hierarchy can be altered experimentally. The established dominance hierarchy of paired rhesus monkeys can be reversed when the dominant monkey of a pair undergoes avoidance conditioning with his submissive partner as the conditioned stimulus (Miller et $\underline{\text { al. }}$. 1955; Murphy \& Miller, 1956). After avoidance conditioning, the submissive monkey shows little dominant behavior but now elicits submissive behavior from the formerly dominant monkey. Avoidance conditioning can change a dominant monkey into a submissive one, but it is questionable whether a submissive monkey can be conditioned to show dominant behavior. 
of tranquilizers (Leary \& S1ye, 1959; Leary \& Stynes, 1959). Meprobamate and chlorpromazine were administered to the dominant rhesus monkey of a pair before SD testing. Analysis of the aggressive acts between the pair indicated the drug treatment had little or no effect on the existing SD relationship. The dominant monkey, although tranquilized, still elicited fear and submissive behavior from the normal1y submissive partner. It may be naive to assume that a we11-established SD relationship can be reversed in on $1 \mathrm{y}$ ten testing sessions by partially tranquilizing the dominant monkey and without manipulating the submissive monkey's learned fear of the dominant monkey. The avoidance conditioning studies indicate that to change SD it is important for the dominant animal to begin displaying submissive behavior and this change does not occur in the tranquilized monkey.

Manipulations of endocrine levels have yielded results similar to the tranquilizer studies. Attempts to alter SD in rhesus monkeys or chimpanzees with hormone treatment of either estrogen or androgen have been unsuccessful (Birch \& Clark, 1950; Mirsky, 1955). Sex-skin swelling must be controlled for in hormone studies. In the chimpanzee but not in the rhesus monkey, an increase in SD accompanies sex-skin swelling and no change in SD occurs with hormone treatment if the sex-skin swelling is inhibited.

In contrast to the negative results of the drug and hormone studies, brain lesions or stimulation can markedly alter the SD hierarchy. With regard to brain lesions and SD behavior, a lesion of the prefonta1 cortex (Brody et a1., 1952) has an effect on SD opposite to that of a temporal lobectomy (Rosvold et al., 1954), . 
while a cingulate cortex lesion (Mirsky et al., 1957) has little or no effect.

In the Brody et al. study, rhesus monkeys first, fifth and sixth (last) in dominance were given prefrontal lesions. The monkey first in SD showed no change in social primacy, while both monkeys fifth and sixth in SD showed increased aggression to monkeys higher in the social hierarchy and decreased responses to the threats of other dominant monkeys. There also resulted an intensification of aggression by high ranking non-operated monkeys and an instability in the behavior of the colony. It appears that prefrontal lesions destroy the submissive monkey's ability to respond appropriately to threat behavior and prevent the lesioned monkey from utilizing feedback from aggressive interactions.

In contrast, dominant monkeys with temporal pole-amygdala lesions show a decrease in SD (Rosvold et al., 1954). This study will be discussed in the next section.

Lesions of the anterior cingulate cortex have no effect on the SD status of either the most or least dominant monkey in a group. The lesioned monkeys show only a temporary fearlessness of man and no change in group social behavior (Mirsky et al., 1957). Complementing the lesion work is the data from brain stimulation. SD has been studied following remote stimulation of the brain in a task free colony of rhesus monkeys (Delgado, 1963, 1964, $1965 \mathrm{a}, 1965 \mathrm{~b})$. Stimulation of the central gray or posterovental nucleus of the thalamus in the dominant monkey evoked increased aggression which was conditioned to the sound of a tone. Stimulation of the caudate nucleus of the dominant monkey inhibited his 
aggression and a submissive monkey learned to push a lever which stimulated the boss' caudate nucleus (cerebral heterostimulation). Another interesting result was that a monkey's response to brain stimulation, whether dominant or submissive, depends upon in position in the SD hierarchy of that particular colony. It appears that stimulation of the same brain structure under different environmental conditions results in different behavioral patterns. 
THE ROLE OF TEMPORAL LOBE STRUCTURES IN AFFECTIVE AND SOCIAL BEHAVIOR

SD can be defined in terms of a series of dominant-submissive relationships that have components of fear, rage, aggression, and placidity. Stimulation or ablation of the amygdala has been investigated in many species, and changes in each of the above affective components has been reported (Goddard, 1964). It is therefore logical to predict that an amygdalectomy would influence SD.

Anatomicarly, the amygdaloid nuclei can be divided into corticomedial and basolateral cell groupings located within the inferior aspect of the temporal pole. The corticomedial group, which phylogentically is first seen in tailess amphibians, is evolutionarily older than the basolateral group. The basolateral complex appears first in reptiles. In higher species there is a gradual reduction of the corticomedial complex and a continuing development and differentiation of the basolateral complex, which attains its greatest complexity in man (Gloor, 1960). Structurally, the amygdala is a component of the basal ganglia, and functionally, it is part of the limbic system.

The amygdala has diffuse afferent and efferent connections. The amygdala's afferent connections include the lateral olfactory bulb, pyriform cortex, inferior temporal gyrus, temporal pole, brain-stem reticular formation, cerebellum, hippocampus, striatum; 
thalamus, hypothalamus, and all the various sensory modailities (Goddard, 1964).

On the efferent side, Nauta (1961) has identified two major amygdala-subcortical fiber systems--a massive, relatively diffuse ventral amygdalofugal pathway and the compact stria terminalis. The stria terminalis projects to the septal region, preoptic area, anterior hypothalamus, ventromedial hypothalamus, and bed nucleus of the stria terminalis. The ventral amygdalofugal pathway projects to the lentiform nucleus, substantia innominata, lateral preoptic and hypothalamic regions, nucleus of Broca's diagonal band, olfactory tubercle, dorsomedial thalamic nucleus, subcallosal gyrus, and rostral cingulate cortex. There are also efferents to rostral parts of the superior, middle and inferior temporal gyri, ventral insular cortex, claustrum, caudal orbito-frontal cortex and contralateral amygdala via anterior commissure. Although the amygdala and hippocampus are juxtaposed, there is yet no anatomical evidence of direct amygdala-hippocampal connections (Nauta, 1961). The above anatomical connections correspond roughly to the primary projection areas determined by electrophysiological methods. The secondary projection field, characterized by longer latency multisynaptic connections, consists of the remainder of the hypothalamus, subthalamus, entopeduncular nucleus, mesencephalic tegmentum, hippocampus, diffuse thalamic projection system and ultimately most of the neocortex (Gloor, 1960).

The amygdala not only has diffuse connections with the limbic system, but also with all of the major subcortical nuclei. The diffuse connections of the amygdala are reflected in microelectrode 
work (Sawa \& Delgado, 1963) which shows that there is convergence from many modalities on the same amygdaloid celt. The amygdala has been reported to modify the activity of the reticular system (Adey et al., 1960) and, like the reticular system, the amygdala may be important in direction and maintenance of attention with respect to relevent stimuli (Wendt et al., 1962).

Attempts have been made to find behavioral functions topographically represented in the amygdala (MacLean \& Delgado, 1953; Wood, 1958; Gloor, 1960). Although topographical maps have been constructed from stimulation data, Gloor concluded that a comparison of these findings failed to reveal any consistency in localization of various amygdaloid stimulation responses. Functional representation in the amygdala appears to be of a more global and undifferentiated type.

Goddard (1964) has compiled the following list of amygdaloid functions which have been noted in the rat, cat, lynx, agouti, dog, phalanger, rabbit, monkey and man. Stimulation of the amygdala can result in attending, sniffing, chewing, salivation, piloerection, vocalization, flight, rage, fear, pupil dilatation and autonomic changes in respiration, heart rate and blood pressure (either an increase or decrease) and an increase in blood sugar level.

Removal of the amygdala can result in hyperactivity, transient decrease in food intake followed by hyperphagia, hypersexuality, disruption of maternal behavior, fearlessness, loss of aggression, placidity, or less frequently rage and a fall in SD. The amygdala also effects the endocrine system, for removal of the amygdala early in life results in impaired growth and atrophy of the thyroid, 
pituitary, adrenal and pancreatic glands. Lesions in the adult animal reveal testicular degeneration and increased levels of adrenal corticoids in the blood. It is possible that some of the emotional changes after amygdalectomy have a basis in the disrupted endocrine system.

With regard to leaming, amygdalectomized animals generally show slower acquisition, more rapid extinction, almost normal or deficient retention of an active or passive, simple or complex, conditioned avoidance response (Weiskrantz, 1956; Pribram \& Weiskrantz, 1957; King, 1958; Horvath, 1963; Robinson, 1963). Amygdalectomized monkeys do not show normal shifts in response to increased or decreased food reward (Schwartzbaum, 1960). They show an impairment on transposition along a brightness continuum (Schwartzbaum \& Pribram, 1960), on transfer of training (Bagshaw \& Pribram, 1965) and on learning-set and discrimination reversal problems (Schwartzbaum \& Poulos, 1965). Because some amygdala lesions include various parts of the temporal lobe, it should be noted that lesions of the inferior and superior temporal gyrus produce deficits in visual and olfactory discrimination (Pribram \& Mishkin, 1955; Brown et al., 1963; Pinto-Hamuy et al., 1957; Mishkin, 1954).

In contrast to the above results, amygdalectomy does not affect postoperative learning of either visual (size, brightness, pattern) or somesthetic (roughness, size) discrimination, although the lesion produces mild retention deficits in visual pattern discrimination acquired preoperatively. On the basis of these negative results, Schwartzbaum (1965) concludes that there is no evidence for disturb- 
ance in simple visual or somesthetic perceptual function following amygdalectomy, and there is little evidence that the bizarre symptoms of the amygdaloid syndrome derive from a general deficit in the learning process.

At the level of simple discriminations and perceptual tasks, Schwartzbaum's reasoning is correct. But at the level of cifficult discriminations and perceptual tasks, the amygdalectomized animal does show perceptual and discrimination deficits which may contribute to the disruption of the monkey's emotional behavior. In the final evaluation of the amygdala's function, learning deficits should be considered along with the emotional changes characteristic of this lesion.

Following an amygdalectomy, the emotional changes commonly seen are fearlessness, tameness, docility and placidity. These emotional changes would certainly influence the SD pattern of a dominant monkey. Because of the vast literature on the amygdala, only papers relevant to these emotional changes will be reviewed, with special emphasis on primate data.

\section{Emotional changes following amygdalectomy}

In 1937, Kluver and Bucy removed the temporal lobes, including amygdala and part of the hippocampus, from rhesus monkeys. They described changes in behavior now referred to as the Kluver-Bucy syndrome. These temporal lobotomized monkeys exhibited visual agnosia, hypermetamorphosis or intensified attention and reaction to visual stimuli, oral compulsive behavior, hypersexuality, changes in dietary habits wich acceptance of meat as food, increased and peculiar spontaneous motor activity, and, of particular interest, profound changes 
in emotional behavior with loss of fear and aggressiveness. For example, an operated monkey, after being bitten and attacked by another animal, may repeatediy approach this animal to examine it. This placidity, lack of aggression, or fearlessness following temporal lobe lesions has been reported for agoutis, lynx, monkeys, cats (Schreiner \& Kling, 1953), rats (King, 1958), Norway rats (Wood, 1956), hamsters (Bunnell et al., 1965) and man (Terzian \& Ore, 1955).

The appearance and permanency of fearlessness and other components of the Kluver-Bucy syndrome seem to depend on the lesion size and amount of amygdaloid involvement. Of all the changes accompanying temporal lobe-amygdala lesions, fearlessness, oral exploration and visual agnosia are the most reliable and consistent, while hypersexuality and dietary changes are more often transient effects or absent. The size and locus of lesions that have been made vary from restricted temporal cortex to temporal lobotomies. Complete bilateral removal of neotemporal cortex, sparing cortex medial to rhinal fissure, (Akert et al., 1961) produced tameness, lack of fear, oral exploration and psychic blindness, no hypersexuality, and all the se effects were markedly decreased after three months. Histology showed fiber degeneration to the amygdala and five of the nine monkeys had minimal direct amygdala damage. Looking at other cortical lesions, no effects have been reported following bilateral lesions of the first or second and third temporal convolutions (Kluver \& Bucy, 1939), incomplete temporal neocortical lesions (Walker et al., 1953), and resection of the inferior temporal convexity (Weiskrantz, 1958). Thus, if temporal neocortical lesions are not complete, the Kluver-Bucy syndrome is absent, if the neocortical 
lesions are complete, the Kluver-Bucy changes which do appear decrease after three months.

Lesions which include most of the amygdala, or amygdala and immediately adjacent temporal area, usually result in at least some of the Kluver-Bucy components. Walker et a1. (1953) found a general correlation between damage to the amygdala area and tameness but no correlation between temporal cortical or hippocampal damage and tameness. The fact that they did not find oral exploration, visual agnosia and only little sexual and dietary changes may be due to the small size of their lesions. They do report that tameness in two monkeys, although decreased from immediate postoperative level, persisted for 19 months. In the original Kluver-Bucy monkeys, the changes reported were essentially present after two years. Tameness, loss of fear and docility have been reported for different amygdalahippocampal combination lesions (Anand et al., 1959; Orbach et al., 1960) but these observations were made over only a two month period. The only study attempting to give experimental meaning to the concept of tameness or fearlessness is that of Weiskrantz (1958). Six monkeys sustained medial temporal pole and bilateral amygdalectomies ( $\mathrm{AM}^{\prime} \mathrm{s}$ ); six with bilateral resection of the inferior temporal convexity and six with a sham operation. Postoperatively, there was an immediate difference between the AM's and the two other groups. The AM monkeys permitted petting, handing, and showed a disappearance of the fear responses while controls continued their usual. aggressive behavior. Visual exposure to a human face was used during panel pressing for food to test for conditioned depression. The same stimulus was also used as the US in a conditioned avoidance 
task. In contrast to the marked fear response of the controls, AM monkeys responded with little or no fear to the human face used as the CS in conditioned depression or US in conditioned avoidance.

Although fearlessness or tameness is a common report, several experimenters report that if amygdalectomized monkeys are subjected to tail pinching (Walker et al., 1953); physical restraint (Akert et al., 1961) or handling (Downer, 1962), the lesioned monkeys can react with well directed aggression. It is also reported that amygdalectomized dogs (Fuller et al., 1957) or hooded rats (Bunnel1, 1966), will fight if attacked, although they do not take the initiative. These results suggest an increase in aggressive threshold after amygdalectomy. Not supporting this hypothesis are reports that amygdalectomized monkeys, although bitten and attacked, repeatedly investigate the attacking monkey (Kluver \& Bucy, 1939) and formerly dominant monkeys now attacked till blood flows, show no retaliation (Rosvold et al., 1954). There is no unitary concept to explain all the present data.

There has been a remarkable interspecies replication of tameness following amygdalectomy. The question arises as to the effect of an amygdalectomy at the human level.

Terzian and Ore (1955) report the effects of a bilateral temporal lobotomy in man. The patient was a 19-year-old boy suffering from psychomotor and grand mal seizures with periods of very aggressive behavior, as illustrated by his attempt to crush his younger brother under his feet. EEG recordings indicated epileptogenic foci in both temporal lobes. To correct these symptoms, the 
left temporal lobe, uncus and hippocampus were removed. No improvement occurred in his seizures and his aggressive behavior increased. Subsequently, his right temporal lobe uncus and hippocampus were removed 19 days after the first operation. The classical Kluver-Bucy syndrome was noted in this patient soon after the second operation. Fifteen days after the operation he showed a strong tendency to approach all people who passed near him and to take possession of all objects surrounding him. The oral tendencies did not appear, but the patient demonstrated an insatiable appetite. The patient's language became poorer and eventually was composed of only very elementary sentences concerned with his basic needs. Hypersexuality was noted when the patient displayed, to the doctors, spontaneous erections followed by masturbation and orgasm. Al1 these responses were present after two years. The most surprising change was the complete loss of emotional behavior. The patient no longer experienced the preoperative rage reactions, but showed only indifferent behavior toward the doctors and his parents. On the basis of this study it is possible to conclude that the Kluver-Bucy syndrome which appears in man is remarkably similar to that seen in infrahuman primates.

In contrast to the majority of amygdala studies, Spiegel et al. (1940) and Bard and Mountcastle (1947) reported that after bilateral amygdalectomy placid cats became savage animals which reacted violently to trifling stimuli. This is not a species difference, since placidity has been reported in the cat after amygdalectomy (Schreiner \& Kling, 1953; Anand et al., 1959), nor 
can this savageness be attributed to onset of hypersexuality, since Spiegel's cats were savage immediately. Green et al. (1957) attribute the discrepant results to interference with the blood supply of the anterior hippocampus, which may produce savageness, while similar vascular disturbances in basal ganglia may result in apathy resembling placidity. Green et al. have the only plausible explanation to date.

In summary, it can be said that in species from rodent to man, lesions involving the amygdala generally result in docility or tameness or fearlessness and various other components of the original Kluver-Bucy syndrome such as hypersexuality, oral exploration and a change in diet. Generalizing these changes to the SD situation, the prediction is that the dominant animal, following amygdalectomy, would lose its aggressiveness and lose its dominance. A loss in SD following amygdalectomy has been reported for primates (Rosvold et al., 1954), dogs (Fuller, 1957) and hooded rats (Bunne11, 1966).

Looking at the primate data, Rosvold et al. (1954) first measured the SD hierarchy among eight rhesus monkeys by observing the animals for one hour, twice a day, while peanuts or food pellets were introduced into the group cage. After a dominance hierarchy was established the dominant animal underwent a bilateral amygdalectomy and temporal tip resection. When this animal was tested two weeks postoperatively in the group cage, he fell to the bottom of the hierarchy--eighth in eight monkeys. When this animal was tested in an individual cage, postoperatively he was less fearful of being hand fed by the experimenter. The second animal in the 
original hierarchy then became the dominant monkey and also underwent a bilateral anygdalectomy. When this monkey was reintroduced into the group cage, he too fell in SD and was now seventh in eight monkeys. Finally, the third monkey in the original hierarchy, now dominant, underwent the same operation. This monkey did not lose her dominance in the group cage, but like the two previous monkeys, did show less fear in the individual cage of being hand fed by the experimenter.

There are possible anatomical and behavioral explanations for why two animals fell in SD while the third operated animal remained the same. With regard to the location of the lesion, the degree of SD change in the group cage situation was consistent with the extent of damage to the basolateral nuclei--the third animal sustained little damage to these nuclei and showed no change in SD. With regard to behavioral variables, the first two animals, when reintroduced into the group cage, were confronted with aggressive, competitive monkeys. The third monkey, when postoperatively returned to the group cage, was not confronted with aggressive competitors nor did she change in SD. Postoperatively, all three animals were less fearful in the individual cage of being hand fed by the experimenter. Rosvold et al. refer to this behavior as being either "aggressiveness" or "fearlessness" and finally call it aggressiveness. In view of the literature, fearlessness would be a more correct term.

\section{$\underline{\text { Problem }}$}

The present status of the weasurement and manipulation of SD and the effects of temporal lobe lesions on emotional behavio: 
and social dominance have been reviewed. There were two basic questions that the present investigation attempted to answer. Do squirrel and cebus monkeys display a measurable SD? What is the effect of a bilateral temporal lobectomy on the squirrel monkey's SD behavior?

The first question arises from data on the rhesus monkeys indicating that these monkeys have a straight-line SD hierarchy, which, once formed, is almost permanent. If a similar SD structure is found in squirrel and cebus monkeys, the concept of SD receives a broader basis for generalization from this comparison across intraorders. In addition, the squirrel monkey, a New Horld lissencephalic monkey, is more primitive in anatomical development than the 01d World gyrencephalic rhesus monkey. The cebus have a more highly convoluted cortex than squirrel monkeys and fall between squirrel and rhesus monkeys on complex behavioral tasks. What effect has this intraorder evolutionary difference exerted on a behavioral phenomenon such as SD?

The second question has its basis in the studies on emotional changes following temporal lobectomies and in the single study exploring the influence of a temporal lobectomy on primate SD (Rosvold et al., 1954). Animals with temporal lobectomies are reported to be tame, docile, fearless, less aggressive, and less dominant. What is the effect of a bilateral temporal lobectomy on the squirrel monkey's SD behavior? A number of specific questions can be formulated. First, can the fall in SD noted in Old World rhesus monkeys following temporal lobectomy be generalized to New World monkeys? If squirrel monkeys show the same 
behavioral changes following temporal lobectomy as reported in rhesus monkeys, there is then evidence for similar brain structures in different species serving the same behavioral functions. Second, what behavioral changes are associated with a dominant monkey's fall in SD and how permanent are these changes? Third, are the effects of a temporal lobectomy on SD identical under positive and negative reinforcing contingencies? There is evidence that animals with temporal lobe and amygdala lesions show a decrement in learning and rapid extinction with negative reinforcement (Brady et al., 1954; Weiskrantz, 1956; Pribram \& Weiskrantz, 1957; King, 1958). From the learning data it might be predicted that the effects of temporal lobectomy on SD would be different as the result of the motivational differences in reinforcement contingencies. 


\section{EXPERIMENT I}

SD HIERARCHY IN THE SOUTH AMERICAN SQUIRREL MONKEY

AND COMPARISON WITH REPORTS ON THE RHESUS MONKEY

In order to assess the SD hierarchy of the squirrel monkey, a series of behavioral tests were developed. These tests were designed so that the monkeys were tested as a group to allow maximum social interactions. In addition, the animals were tested under different experimental conditions and reinforcement contingencies. By employing multiple tests, the probability of measuring the squirrel monkey's SD hierarchy was increased and comparisons could be made across the various testing conditions.

Part 1: Positive Reinforcement

\section{Method}

\section{Subjects}

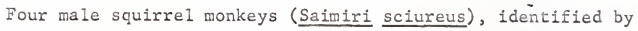
plastic color-coded collars (white, green, red and blue), were used throughout this study. The monkeys weighed between 2 and $2 \frac{1}{4}$ pounds and were judged to be adults from $X$-rays showing dentation to be complete and epiphyses to be fused. The monkeys were housed as a group in a large cage, $2 \frac{1}{2} \times 3 \times 3$ feet, two weeks before testing began and throughout the sixteen months of testing. During positive reinforcement testing the monkeys were fed separately and maintained on a deprivation diet of 80-90 per cent body weight. During negative reinforcement testing the monkeys were fed together ad 1 ib. 
The four monkeys represented two varieties of squirrel monkeys. White, Green and Red had a circumocular patch that was light in color and formed a peak similar to a Gothic arch. In contrast, Blue's eye patch was darker and rounded, in the manner of a Roman arch. Accordingly, these two types have been named Gothic and Rowan. The Gothic type is probably Colombian or Peruvian in origin whereas the Roman type is probably native to Brazil (MacLean, 1964).

\section{Apparatus}

The apparatus consisted of two $2 \times 2 \times 2$ feet compartments connected by a narrow plexiglass tunnel seven inches in length, which allowed only one monkey to cross at a time. To facilitate observation, the front and one side of each compartment was plexiglass. A removable food cup was placed on the wall of each compartment opposite the entrance to the tunnel, and was visible from the other compartment. The floor of each compartment and the tunnel consisted of a grill of brass rods attached to a shock scrambler. A guillotine door on each side of the tunnel controlled access to either compartment. A buzzer was mounted on top of the apparatus and a light in each compartment could be flashed at a variable rate.

$\underline{\text { Procedure }}$

Two weeks after the four monkeys had been placed in the group cage, individual training in the shuttle apparatus began. The monkeys were placed singly in one compartment, and the experimenter baited the food cup in the opposite compartment with currants or dextrose pellets were dispensed automatically. The buzzer was 
sounded, and two seconds later the door opened, allowing access to the opposite compartment and food cup. This training was continued at the rate of 20 trials per day until each animal reached the criterion of responding within five seconds of the door opening for 18 of the 20 trials. Following individual training the four monkeys were tested together in the shuttle box. The trial procedure was identical, but between trials seven social interactions (pushing, pulling, biting, attacking, chasing, mounting and genital display) were recorded or two minutes were permitted to elapse before beginning the next trial. Those monkeys which crossed the tunnel to obtain food usually rejoined the other monkeys which had not crossed the tunnel. If the four monkeys did not reassemble in one compartment, the experimenter walked toward one of the compartments and his approach elicited escape behavior to the desired compartment. Fifteen trials were run each day, and required a total of about 30 minutes. If any monkey did not obtain five currants in the 15 trials, he was individually given five trials at the end of group testing to control for the effects of practice and extinction of the shuttle response. On alternate days, the four animals were tested in their home cage. In this procedure a buzzer was sounded and three currants were dropped into a food box. No individual training was given to the monkeys on this home cage task. Seven social interactions were recorded or two minutes were permitted to elapse before beginning the next trial. Testing in the home cage and shuttle box permitted comparison of results from a large versus restricted space and living cage versus experimental apparatus. Following group testing for 12 sessions, which alternated between 
shuttle box and home cage, the animals were tested after sequential removal of the most dominant monkey, next dominant, etc. When this testing procedure was completed, the animals advanced to Part 2.

\section{Scoring procedure}

The same scorizg procedure was used for home cage and shuttle box testing. In the shuttle box, the order of the monkey's crossing through the tunnel to obtain food (or avoid shock) was intended as a possible adjunct to the primary measure of the existing social hierarchy. The primary measure of SD was taken between competitive trials, during the intertrial interval. During this period, constant aggressive interactions occurred and the frequency of these behaviors (pushing, pulling, biting, attacking, chasing, mounting and genital display) were recorded, scoring one for each aggressive interaction and two for each aggressive chase. The total number of aggressive responses (pushing, pulling, etc.) a monkey initiated represented his aggressive score. The submissive score was computed by counting the number of aggressive responses a monkey received. Squirrel monkeys were rarely observed to make submissive gestures, therefore, a monkey that was consistently attacked was defined as showing submissive behavior. Thus, trials for food or shock avoidance competition resulted in aggressive social interactions, which were expressed and measured during intertrial intervals. Priority for incentives was not the index of SD.

\section{$\underline{\operatorname{Resu} 1 \mathrm{ts}}$}

As shown in Figure 1, the SD hierarchy for the adult male 
squirrel monkey was both linear and stable. It is obvious that White was the dominant animal with the other three animals, Green, Red, Blue, following in that order. Behavior in the home cage closely paralleled the shuttle box with only a change in the order of the social hierarchy. The order of the monkeys' crossing through the tunnel clearly revealed the dominant animal (White crossed first 60 times out of 90 trials) but did not distinguish between the SD of the other three animals. Another indication of dominance was the observation that if White crossed first through the tunnel to obtain food, rarely did another monkey follow White, and if a monkey followed, it never competed with White for the food. But if Green, Red, or Blue crossed first, White always followed and competed with the less dominant animal. White was the only monkey to actively keep the other monkeys away from the area around the guillotine door. To determine whether the social hierarchy was linear, the animals were subsequently tested in the following groups: Green, Red and Blue (White removed); and Red and Blue (White and Green removed). In both situations linearity was maintained. The final social hierarchy, determined from combining all above measures, was consistently White, Green, Red and Blue.

\section{Discussion}

The observation of a linear social hierarchy among squirrel monkeys has not been previously reported and differs in part with the conclusions of Ploog et al. (1963) who questioned linearity in the social hierarchy of the squirrel monkey. A linear social hierarchy has been consistently reported for the rhesus monkey (Miller 
and Murphy, 1956; Miller and Banks, 1962). The present study suggests that the adult male rhesus and squirrel monkeys may have a similar social structure and organization. The present observation of a linear social hierarchy in the squirrel monkey may result, in part, from employing a combination of measures rather than a single measure.

Part 2: Negative Reinforcement

\section{Method}

\section{$\underline{\text { Procedure }}$}

At the end of Part 1, the monkeys were tested for the effects of negative reinforcement on their SD. The monkeys were individually trained to avoid shock by crossing from one compartment of the previously described apparatus to the other at the onset of a flashing light (CS). When each animal had reached a criterion of 18 avoidance responses in 20 trials, by crossing within five seconds after door raised, the monkeys were tested together. For group testing in the shuttle box, a trial consisted of two seconds of flashing light followed by the door opening. Shock began as soon as the first monkey had moved from one compartment to the other. Thus, the first animal through the tunnel always avoided shock, while the other three could escape from the shock. In this manner competition was maintained among the monkeys. Fifteen trials were given a day. As an additional measure of SD, on alternate days, the monkeys were observed without experimental manipulation in their home cage for 30 minutes, and social interactions were recorded. 


\section{$\underline{\text { Results }}$}

The social kierarchy under negative reinforcement was very similar to that displayed under positive reinforcement. With negative reinforcement, as seen in Figure 1, the hierarchy was slightly less stable and linearity was not quite as absolute as. in the positive situation. Again, there were changes in frequencies of responses between the home cage and the shuttle box, but as in the positive situation, no change in hierarchy occurred. The order of crossing through the tunnel in the negative situation partially correlated with the existing social hierarchy. White crossed first most frequently (40), Green crossed second most frequently (41), Blue third (33) and Red last (55). The social order of Red and Blue was reversed here. The explanation of this apparent shift lies in the fact that as soon as Red crossed, Green would attack Red. As a result, Red remained in the electrified compartment and received the shock in preference to exposing himself to Green's attacks.

\section{Discussion}

The nature of reinforcement in a test situation may have important consequences for an animal's social behavior. Green (second in dominance) developed the response of sitting on or mounting Red for up to five minutes and often a "tower" would form with Red on the bottom, Green above him and White on top. The tower was seen only in this testing situation. In the negative situation White (dominant animal) was less active, but still dominant. Green was more active in the negative situation and this change in activity was the reciprocal of White's decrease 
in activity. Each animal consistently directed most of its aggressive attacks toward a particular animal (White against Green; Green against Red; Red against Blue). A decrement in the activity of one monkey produced a release of aggressive behavior in other anicials. The generalization of a social hierarchy across reinforcement contingencies has also been reported in the rhesus monkey (Hamilton, 1960; Miller and Banks, 1962). The results of this study agree with these investigators and point to an apparent similarity between the social structures of these two species.

Part 3: Retesting with Positive Reinforcement

After the monkeys had completed the negative reinforcement sequence, they were retested on positive reinforcement to assess any changes in the SD structure resulting from exposure to the negative reinforcement situation.

\section{$\underline{\text { Procedure }}$}

The four monkeys were individually tested for retention of the shuttle response with food reinforcement. The four monkeys were then tested together altemating between the shuttle box and home cage for two sessions each.

\section{$\underline{\text { Results }}$}

A11 monkeys reached criterion on the shuttle response. As seen in Figure 2, when the monkeys were tested as a group, the SD hierarchy in both shuttle box and home cage was unchanged. From most to least cominant the order was White, Green, Red and Blue. Thus, the monkey's SD hierarchy, observed under positive reinforcewent and after exposure to negative reinforcement, was identical to the hierarchy initially observed under positive reinforcement. 
Part 4: SD Hierarchy after Two-week Separation

It was necessary after anterior temporal lobectomy to allow the monkey a two-week recovery period, during which time the four monkeys were housed in separate cages. Thus, it was necessary to test for any change in the normal monkey's SD hierarchy which might result from a two-week separation period.

\section{Procedure}

The four monkeys were separated and housed in individual cages for two weeks. After two weeks the four monkeys were individually tested for retention of the shuttle response. The next day group testing was begun with the four monkeys tested under positive reinforcement. The shuttle box and home cage tests were alternated for three sessions each.

\section{Results}

As shown in Figure 3, the two-week separation period did not alter the previously established SD hierarchy. White was still the dominant monkey followed by Green, Red and Blue. It was concluded that the postoperative separation period would not influence the established SD hierarchy. Part 5: Reaction to a Fearful Stimulus and to the Experimenter A temporal lobectomy is reported to increase an animal's approach to novel and noxious stimuli. In order to compare the normal and temporal lobectomized monkey's approach behavior, a series of tests evaluated the normal animal's response to fearful objects (test for visual agnosia). The monkeys were hand fed by the experimenter and resistance to attempted capture with the experimenter's gloved hand was evaluated. These tests measured affect independent of the SD testing procedure. 


\section{SQUIRREL MONKEYS}

\section{POSITIVE REINFORCEMENT NEGATIVE REINFORCEMENT}

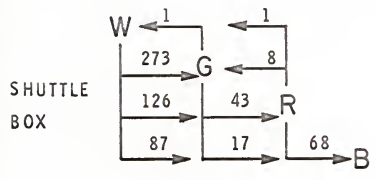

TOTAL 624

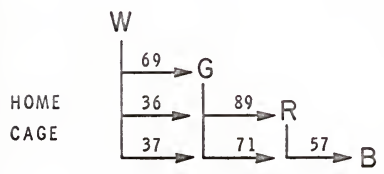

TOTAL 360

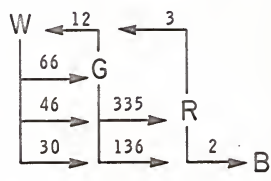

TOTAL 629

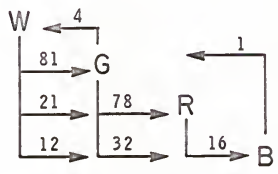

TOTAL 246

Figure 1. Frequency of responses (pulling, pusning, biting, attacking, chasing, mounting, and genital displayl recorded during the intertrial interval. The letter indicates the monkey, the arrow indicates the direction of aggression and accompanying number indicates frequency of aggressive responses. 


\section{SQUIRREL MONKEYS \\ RETEST AFTER NEGATIVE REINFORCEMENT}

SHUTTLE BOX

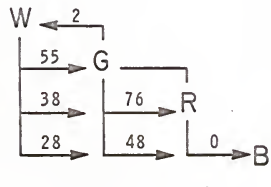

TOTAL 297
HOME CAGE

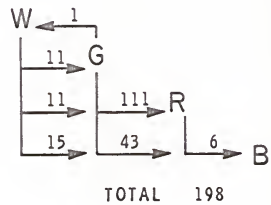

Figure 2. Frequency of responses (pulling, pushing, biting, attacking, chasing, mounting, and genital displayl recorded during competition for positive reinforcement after completion of negative reinforcement sequence. Data represent two test sessions in each situation. The letter indicates the monkey, the arrow indicates the direction of aggression, and accompanying number indicates frequency of aggressive responses. 


\section{SQUIRREL MONKEYS}

\section{AFTER TWO WEEK SEPARATION}

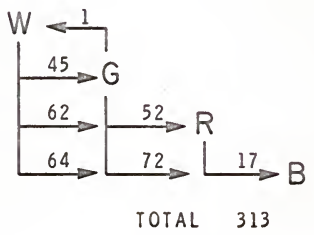

Figure 3. Frequency of responses (pulling, biting, attacking, chasing, mounting, and genital display) recorded during competition for positive reinforcement after monkeys were separated for two weeks. Data for three test sessions in the shuttle box. The letter indicates the monkey, the arrow indicates the direction of aggression and accompanying number indicates frequency of agonistic responses. 


\section{Procedure}

The monkeys were individually tested in the shuttle box with a live cat in the opposite compartment and the tunnel between compartments closed. The time the monkey spent during a five-minute period in front of, or at the back of, his compartment (farthest point from the cat) was recorded. A week later, the monkeys were tested singly with the tunnel open and a second fearful object, a large, colorfully decorated teddy bear, in the opposite compartment. The monkey's position with respect to the teddy bear was recorded for five minutes. After five minutes, the monkey was shocked for three seconds on each of nine trials in an attempt to drive him from his own compartment into that containing the fearful object. If he had not crossed through the tunnel to the bear's compartment by the ninth shocked trial, he was shocked for thirty seconds on the tenth trial. The monkey could escape the shock by crossing into the compartment containing the toy bear and was then free to return to his own compartment until the next trial. The shocked trials measured the strength of the fear and reluctance of the monkey to approach the teddy bear.

To assess the monkey's fear of a human, the experimenter sat in front of one of the compartments and handed food pellets to the monkey. The monkey's position and aggressive behavior was recorded before, during, and after the monkey took a pellet from the experimenter's hand.

The monkeys were also individually tested for resistance to capture with the experimenter's gloved hand. For one minute, the experimenter tried to touch or grab the monkey in the compartment. 


\section{$\underline{\text { Results }}$}

All four monkeys were extremely fearful of the cat and spent an average of four minutes and forty-two seconds of the five minutes in the back of their cubicle, which was the farthest point from the cat. The reaction of the monkeys to the toy bear was identical to their response to the live cat. The monkeys spent an average of four minutes and forty-three seconds of the five minute period in the extreme rear of their cubicle. During the five minute period, all four monkeys were hyperactive and continuously looked at the novel stimulus. None of the four monkeys crossed into the teddy bear's compartment without being forced by shock, although the tunnel was always open. White, the dominant monkey, did not cross even after 10 shocked trials. Blue did not cross the tunnel either, but this result is confounded by a previous accidental encounter and skirmish with the live cat in the shuttle box, which may have augmented his fear of the testing procedure. Green and Red crossed to the teddy bear's compartment after six and five shocked trials respectively. They immediately recrossed to their own compartment without sniffing or contacting the toy bear and maintained this sequence for the remainder of the ten trials. It is interesting that the dominant animal seemed more terrified of the novel stimulus than the other, less dominant, monkeys.

The monkeys showed no fearful or aggressive reactions to the experimenter's presence or hand feeding. Within five trials, all the monkeys sat in front of the cube and waited for the pellets. Because of these negative results this test was eliminated. 
All the normal monkeys showed great resistance to being caught. Only Blue could be touched with the gloved hand. The other three monkeys showed such violent avoidance of gloved hand, that the test was terminated before the monkeys could do physical injury to themselves. White, Green and Red ran around and into the sides of the testing compartment to avoid the gloved hand.

\section{Discussion}

The four monkeys were tested for SD under positive and negative reinforcement, after the monkeys were selectively removed from the group to test for linearity, and after two weeks of isolation. In all cases the SD hierarchy remained linear and stable. The monkeys were then tested for fear responses to novel or noxious stimuli and resistance to capture. Because this testing procedure incorporated different reinforcement contingencies, measured a number of different responses and gave consistent results, this procedure was used to analyze the effects of bilateral temporal lobectomy on the squirrel monkey. 


\section{EXPERIMENT II}

THE EFFECT OF ANTERIOR TEMPORAL LOBECTOMY

ON THE SD BEHAVIOR OF THE SQUTRREL MONKEY

Ia Experiment I, behavioral tests were developed to assess the SD hierarchy of the squirrel monkey. In the present study, Experiment II, these behavioral tests were used to assess the effects of a bilaterally temporal lobectomy on the squirre monkey's previously measured SD hierarchy. Specifically, Experiment II investigated (1) the effects of a temporal lobectomy on the emotional and SD behavior of the squirrel monkey, (2) whether a temporal lobectomy changes a dominant monkey to a submissive one, and (3) the effects of positive and negative reinforcement contingencies on the SD status of a temporal lobectomized monkey. Procedure

Following completion of Experiment I, the dominant monkey was operated and then tested in the sequence shown in Table 1. The testing sequence, apparatus and scoring method were identical with those described in Experiment I. At the time of surgery on the dominant monkey, the remaining three monkeys were separated and placed in individual cages. The monkeys remained separated during the two-week postoperative recovery period, so that there was no opportunity for sub-hierarchies to develop. Ten days after surgery all monkeys were put on a deprivation diet and maintained at 90 per cent body weight throughout positive reinforcement testing. 
TABLE 1

The testing sequence after the dominant monkey was operated

1. Dominant monkey was operated and permitted a two-week recovery period

2. Positive reinforcement sequence--one month duration

3. Negative reinforcement sequence--one month duration

4. Retesting with positive reinforcement--two weeks duration

5. Reaction to a fearful stimulus--one day duration

6. Reaction to being handled--one day duration

7. Animals separated, next dominant monkey operated and sequence repeated in above order 
Two weeks after operation, each monkey was tested individually for retention of the shuttle response under both positive and negative reinforcement. If all monkeys reached the criterion $(18 / 20)$ they were tested as a group the next day. If a monkey did not reach criterion, it was retrained individually until it met the criterion before group testing began. After the first group testing session, the monkeys again lived together in a group cage.

\section{$\underline{\text { Surgery }}$}

The operation was performed under sterile procedures in a single stage with Nembutal anesthesia $(17 \mathrm{mg} \cdot / \mathrm{Kg})$ and $.2 \mathrm{cc}$ of Atropine. The monkey was held in the supine position by the stereotaxic head holder. A "C" shaped incision was made on the side of his head, exposing the temporal muscle, which was bluntly dissected and retracted to expose the temporal bone. A trephine hole was cut into the temporal bone, the dura incised, and the lesion made by aspiration of the temporal pole, inferior temporal gyrus, and amygdala, attempting to spare the posterior hippocampal formation. When the ablation was completed, it was packed with several small pieces of gelfoam, the facia and muscle were closed in layers, and the operative procedure was repeated on the other side. During postoperative recovery, the animals were given $20 \mathrm{cc}$ of Sustagen orally for the first four days. Some of the monkeys showed a transient aphagia for solid monkey biscuits, but drank Sustagen, licked sugar cubes and ate fruit. After a week the operated animals were eating monkey biscuits. 


\section{Histology}

When all monkeys had completed testing, they were sacrificed and perfused with saline and 10 per cent formalin through the left ventricle. The brains were removed and the gross lesion was reconstructed by projecting photographic negatives of the brain onto brain maps and tracing the lesion. Then the brains were embedded in celloidin and serial, coronal sections were cut at 30 micra. Every tenth and eleventh section was sustained with Weil's stain and cresyl violet, respectively. The lesion was also reconstructed by the use of representative serial sections.

\section{Anatomical results}

The reconstruction of White's lesion is shown in Figure 4 . The anterior and medial temporal pole areas were bilaterally resected. In the right hemisphere, a small portion of the anterior, medial, and corticomedial amygdala remained. In the left hemisphere, a small amount of anterior and corticomedial amygdala was spared. Posteriorly, the hippocampus and dentate gyrus were bilaterally invaded to the level of the lateral geniculate body.

Green's lesion is reconstructed in Figure 5. The anterior and medial temporal pole areas were bilaterally ablated. In the right hemisphere, a strip of corticomedial amygdala remained. In the left hemisphere, a narrow strip of anterior, medial and basolateral amygdala was spared. The posterior extent of the lesion included bilateral damage to the hippocampus and dentate gyrus.

The reconstruction of Red's lesion is shown in Figure 6 . The anterior and medial temporal pole areas were only slightly damaged. In the right hemisphere the amygdala was totally ablated except for 

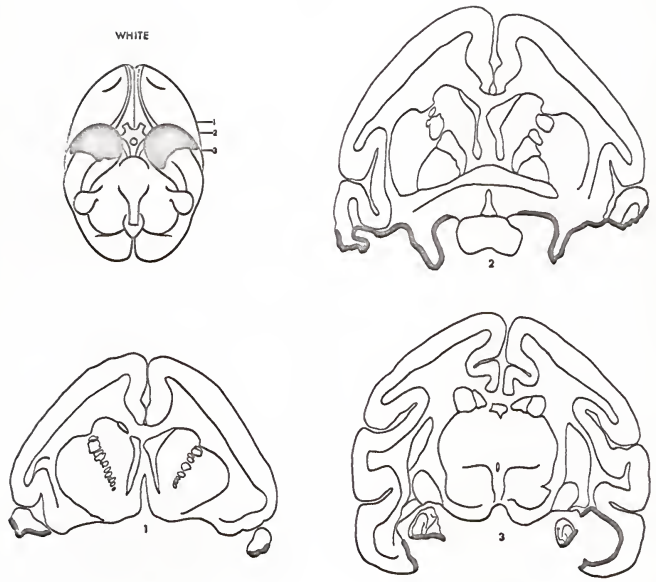

Figure 4. Reconstruction of White's lesion. The dark area on the gross brain map shows amount of damage to the inferior and medial temporal pole area. In the serial sections, the extent of the lesion is indicated by the heavy black line. 

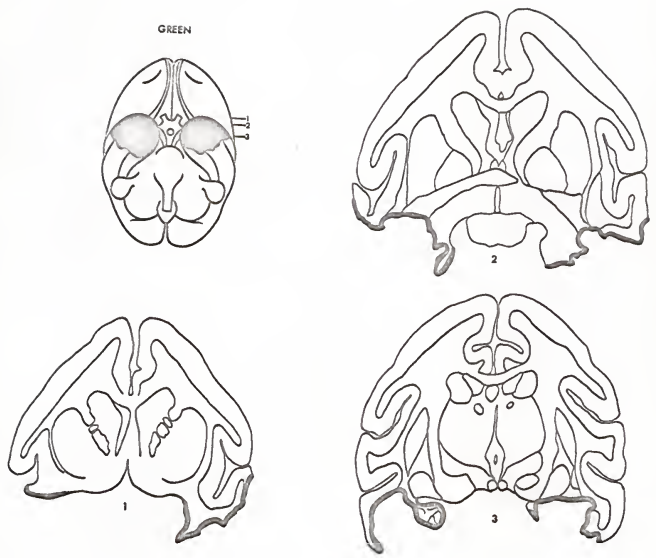

Figure 5. Reconstruction of Green's lesion. The dark area on the gross brain map shows amount of damage to the inferior and medial temporal pole area. In the serial sections, the extent of the lesion is indicated by the heavy black line. 

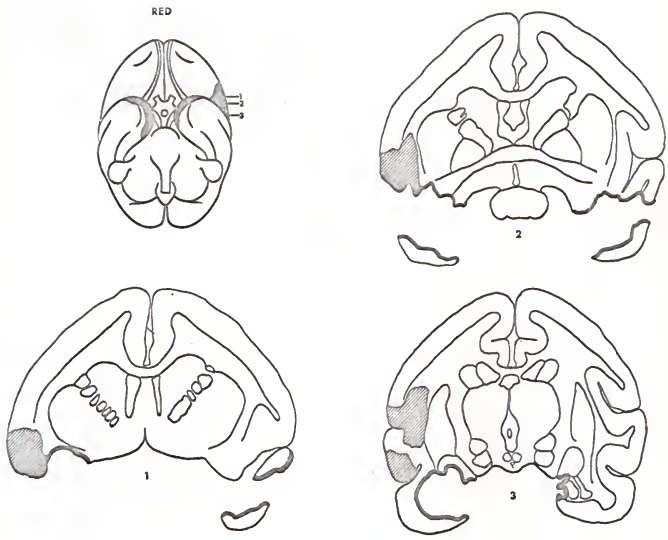

Figure 6. Reconstruction of Red's lesion. The dark area on the gross brain map shows amount of damage to the inferior and medial temporal pole area. In the serial sections, the extent of the lesion is indicated by the heavy black line and the area of neuronal atrophy and gliosis is indicated by the shaded region. 

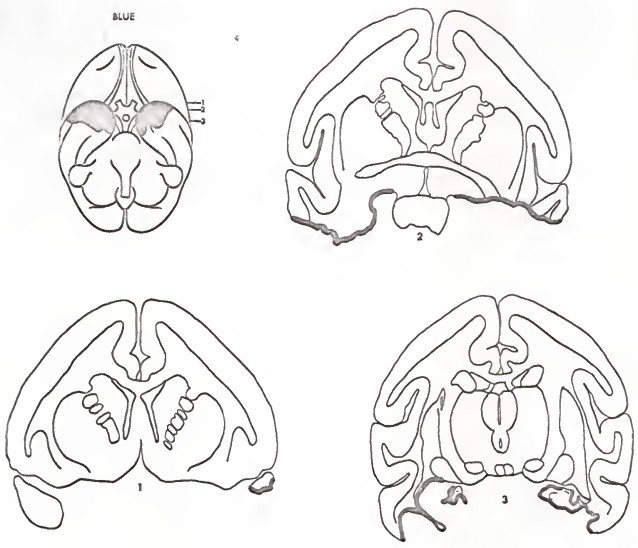

Figure 7. Reconstruction of Blue's lesion. The dark area on the gross brain map shows amount of damage to the inferior and medial temporal pole area. In the serial sections, the extent of the lesion is indicated by the heavy black line. 
a very small part of the medial amygdala. There was some damage to the inferior orbito-frontal cortex. In the left hemisphere, the amygdala was totally destroyed. There was bilateral damage to the lateral optic tracts. Posteriorly, the hippocampus and dentate gyrus were bilaterally invaded. Red also had a right, unilateral temporal and frontal cortical area that showed complete neuronal atrophy and gliosis. This damage was probably caused by interruption of the blood supply to that area.

Blue's lesion is shown in Figure 7. The anterior and medial temporal pole areas were bilaterally resected. In the right hemisphere all amygdala was destroyed. In the left hemisphere, a small area of corticomedial and basolateral amygdala was left. The posterior extent of the lesion included bilateral damage to the hippocampus and dentate gyrus.

The monkeys were ranked for the amount of amygdala removed. Red had an almost total amygdalectomy, Blue only had a small area of amygdala remaining on the right side, while White and Green had about equal amounts of amygdala remaining bilaterally. Red was the only monkey with unilateral inferior orbito-frontal damage, bilateral optic tract damage and tempero-frontal atrophy.

Part 1: Changes Following White's Operation

White was the dominant monkey of the group and the first monkey lesioned.

\section{Positive reinforcement}

The monkeys were individually tested for retention of the shuttle response and all reached criterion. White crossed the 
tunnel into the opposite compartment on the first trial within five seconds after the buzzer was sounded and the door raised, but did not eat the currants in the food cup. After crossing on the second trial, White investigated, sniffed, then ate the currants. On the third trial, White crossed the tunnel and immediately went to the food cup and ate the currants. Because White's postoperative shuttle response appeared somewhat retarded in terms of latency, he was given another twenty trials in the shuttle box the next day, and group testing began the following day.

On the first trial of group testing, White did not compete for currants, showed little aggressive behavior and frequently sniffed the other monkeys. White competed for currants on the fourth trial. In the first group testing session White did not sit next to other monkeys, and avoided monkeys when they sat next to him. Preoperatively, White had consistently and aggressively wedged himself between the other monkeys as they sat side by side between trials. Postoperatively, Green, Red and Blue usually sat next to each other, while White either sat at a distance or momentarily joined the group.

The shuttle box data is shown in Figure 8 . White's aggressive (A) score (total biting, pulling, pushing, attacking, chasing, mounting and genital display responses) decreased to half of the preoperative level $(486-251)$. The scores in parentheses represent the scores before and after White's lesion. White attacked Green less and Green, second in the original SD hierarchy, increasingly attacked Red and Blue. Thus, Green's aggressive score increased from 61 to 341 responses. White showed no submissive behavior. 
It should be noted that the submissive (S) score indicates the number of times a monkey was attacked and does not mean the number of submissive gestures he made to a dominant monkey. Green's submissive score decreased (281-96), Red's S score increased $(169-318)$, as did Blue's $(172-207)$ as a result of Green's increased aggression.

The order of tunnel crossing and food-getting, shown in Figures 9 and 10, indicates that White preoperatively crossed first most frequently and received the most currants (70). Postoperatively, White crossed second most frequently and received less food (42). Green crossed the tunnel first most frequently and obtained the most currants.

In the home cage (Figure 11) White showed almost no decrease in aggressive behavior (142-135), and no submissive behavior. White attacked Green less frequently, but White's A score remained at the preoperative level because White now attacked Blue, who competed with White for currants. Because White attacked Green less, Green attacked Red more frequently (125-319) and Green's A score doubled (160-351).

As seen in Figure 10, White obtained the same number of currants (90), and Blue competed for and received more currants $(6-47)$, as did Green $(10-26)$.

In summary, White showed decreased aggression in the shuttle box, but not in the home cage, and in both situations White's submissive score was zero. White obtained fewer currants in the shuttle box and dropped from first to second in order of tunnel crossing. In the home cage, White obtained the same number of currants. 


\section{Negative reinforcement}

A week after finishing positive reinforcement testing, monkeys were individually tested for retention of the shuttle response and all monkeys reached criterion.

As seen in Figure 12, White's aggressive behavior in the shuttle box had almost disappeared $(142-5)$ and his $S$ score remained the same (15-17). Green's aggressive score increased (483-595) and his $S$ score decreased $(66-3)$ as a result of White's loss of aggression. Red and Blue's aggressive and submissive scores did not change.

The order of tunnel crossing to avoid or escape shock is shown in Figure 13. Preoperatively, White crossed first most frequently and postoperatively, White most frequently crossed last. The new order of tunnel crossing was Blue, Green, Red and White.

The home cage data are shown in Figure 14. White initiated less aggressive behavior (114-36) and Green became more aggressive (114-179). Red and Blue's aggressive scores did not change. White showed little submissive behavior (4-4), and Green showed a decrease in submissive behavior (82-27) because of White's lack of aggression. Blue's S score increased (59-106) and Red's S score remained about the same $(99-115)$.

In summary, White initiated fewer aggressive interactions in the shuttle box and home cage. White showed no increase in submissive behavior in either situation. Green became more aggressive and less submissive due to White's lack of aggression. Green dominated Red and Blue, whose $A$ and $S$ scores changed Ifttle.

\section{New SD hierarchy}

Following White's decrease in aggressive behavior (884-427), 
the SD hierarchy was changed (Figure 15). Green, originally second in SD, now initiated the most aggressive activity (818-1354) and dominated Red and Blue. White continued, but at a markedly lower rate, to attack Red, Blue and Green.

Retesting with positive reinforcement

After testing on negative reinforcement was completed, the monkeys were retested together in the shuttle box and home cage on positive reinforcement. The results from retesting confirmed the results already available from previous testing on positive reinforcement and thus have been omitted.

Reaction to a fearful stimulus and the Kluver-Bucy syndrome

Within two days after operation, White approached, sniffed and licked objects (finger, pen, pliers, ruler) put into or near his home cage. White continued to approach and sniff objects until sacrificed a year after surgery. No hypersexuality--mounting, erections, or genital display--was observed.

As a test for visual agnosia, the lesioned monkey was tested for approach to a teddy bear which the monkey preoperatively avoided. Postoperatively, when White was tested with the teddy bear, he crossed into the bear's compartment and sniffed the bear 15 seconds after being placed in the opposite compartment. As seen in Table 2, White continued to go into and out of the bear's compartment 19 times in the five minute exploratory period. After White had sniffed the bear on his first crossing, he walked around and showed no further exploration of the bear on subsequent entries into the bear's compartment. After the exploratory period, White was shocked once when he was in the neutral compartment, and he immediately 
crossed into the bear's compartment. Preoperatively, White had not crossed into the bear's compartment either during the exploratory period or the ten shocked trials. When the three normal monkeys were tested with the bear, none of them spontaneously crossed into the bear's compartment during the five-minute exploratory period. Green crossed on the first shocked trial, but avoided any contact with the bear, and immediately returned to the neutral compartment. Red crossed on the third shocked trial and, like Green, avoided any contact, returning immediately to the neutral compartment. Blue did not cross into the bear's compartment during the exploratory period or on any of the five shocked trials.

The reaction of the monkeys to being handled with a gloved hand was tested next. Preoperatively, White showed great fear when the gloved hand approached him and he avoided the glove by often running into the sides of the compartment. Postoperatively, however, White showed no fear at the approach of the gloved hand, and sniffed the glove. White did show resistance to being picked up and struggled when held. Normal monkeys, Green and Red, ran into the sides of the compartment at the approach of the glove and testing was terminated. Blue also showed some avoidance of the glove and struggled when held.

In sumary, White showed the following components of the Kluver-Bucy syndrome: sniffing objects, visual agnosia, decrease in aggressive behavior, little licking behavior and no hypersexuality. 


\section{Discussion}

The most dramatic changes shown by White were decreased aggressive behavior, visual agnosia and sniffing behavior. White's aggressive behavior decreased under positive reinforcement and disappeared under negative reinforcement testing. Although this differential decrease in aggression is confounded with order of testing, there is evidence that the decrease in aggression was more marked under negative than under positive reinforcement. Although White became less aggressive, he did not show more submissive behavior (was not attacked more frequently). One reason why White was not attacked more postoperatively may have been that the other monkeys were still responding to White as the dominant monkey. Thus, preoperatively learned dominant-submissive patterns and postoperative behavior were unavoidably confounded. The best description of White's postoperative behavior was that he was passive and reacted to, rather than initiated, aggressive contact. White did react aggressively when monkeys ran over or around him and did attack Blue when Blue competed with him for currants. It appeared as though White's aggressive threshold was raised.

White showed no fear of the teddy bear, the experimenter's presence or glove, struggled and bit the glove when held. After surgery, White did not respond to the experimenter's presence by running from one compartment into the other. In order to chase White into the desired compartment postoperatively, it was necessary to either bang a glove against the side of the compartment or insert a hand inside the compartment. White often sniffed the experimenter's hand. 
Although White sustained extensive bilateral inferior temporal lobe damage, some amygdala was spared bilaterally. White's persisting, although decreased, show of aggression may have resulted from the incomplete amygdalectomy, since the monkey with almost total amygdalectomy (Red) showed no aggression after operation.

Part 2: Changes Following Green's Operation

Before Green's operation all monkeys were changed from currants to dextrose pellets which were automatically dispensed. After one week of testing, the monkeys' shuttle response for dextrose pellets was identical to their performance for currants.

After White's lesion, Green initiated the most aggressive interactions and was the next dominant monkey operated.

\section{Positive reinforcement}

Monkeys were individually tested for retention of the shuttle response and Red and Blue reached criterion. Green made 16/20 shuttle responses and spent much of the testing period exploring the food cup or visually scanning the room. Green reached criterion the next day. White was extremely hyperactive in the shuttle box and although he reached criterion, he did not eat the food pellets for four trials. When retested the next day, White both crossed the tunnel and ate the food pellets.

On the first trial of group testing in the shuttle box, Green did not compete for food and avoided contact with the other monkeys. After the fourth trial Green no longer avoided the other monkeys but sat next to and sniffed them. After the sixth trial Green competed for and obtained food pellets. 
In the shuttle box (Figure 8 ), Green was less aggressive (341-222) and less submissive (96-15). The scores in parentheses represent the scores after White and Green's lesion, respectively. White continued to show less aggression (251-36) and was more submissive $(2-76)$. Red, who was below Green in the original SD hierarchy, displayed a marked increase in aggressive behavior $(32-256)$ and decreased in submissive behavior (318-69). Blue showed no aggressive and more submissive behavior (207-354). Thus, White attacked Green less frequently, Green attacked Red less, and Red attacked Blue frequent1y and White occasionally. As seen in Figures 9 and 10, Green alternated between crossing the tunnel first and second. White most frequently crossed the tunnel first and Red crossed second. White received the most food pellets, Green second, Red third and Blue received the least. In the home cage (Figure 11), Green was less aggressive (351-66) and showed little submissive behavior (15-12). White continued to decrease in aggression (135-31) and increased slightly in submissive behavior (1-26). Red was a little more aggressive (24-43) and strikingly less submissive (319-7), apparently because of Green's lack of aggression. Blue showed minimal aggression $(0-7)$ and was attacked less frequently (175-102). The greatest change in Green's behavior was that he attacked Red less frequently $(272-5)$. As seen in Figure 10, White, Green and Blue obtained about the same number of food pellets ( 80 ) and Red received fewer (42).

In summary, Green showed a marked decrease in aggression in the home cage, although he still initiated aggressive activity in 
the shuttle box. Green did not become more submissive (was not attacked more) in either situation. Green obtained fewer food pellets in the shuttle box and more in the home cage. White's aggressive activity was almost nil in both testing conditions. Red initiated the most aggressive activity, attacking Blue most and White less frequently.

\section{Negative reinforcement}

Monkeys were individually tested for retention of the shuttle response and all reached criterion on the first day. The aggressive and submissive scores in the shuttle box are shown in Figure 12 . Green was markedly less aggressive (545-64) and more submissive $(3-54)$, as Red now attacked Green. White showed little aggressive $(5-25)$ or submissive behavior $(17-26)$. Red was much more aggressive $(5-350)$ and much less submissive $(374-4)$. Blue continued to show no aggression and his submissive score continued to increase (173-364). Red attacked, in order of decreasing frequency, Blue (549), White (82) and Green (79). The order of tunnel crossing after Green's lesion (Figure 13) was the same as that after White's lesion: Blue, Green, Red, with White last.

The home cage data is seen in Figure 14. Green was less aggressive $(179-5)$ and equally submissive $(27-21)$. Red was more aggressive (37-61) and markedly less submissive (115-0), probably because Green attacked Red less frequently. White showed little aggression (36-14) or submission (4-14). Blue initiated no aggression and was attacked less $(106-45)$, as Green was less aggressive.

In summary, Green showed a marked decrease in aggressive be- 
havior in both the home cage and shuttle box. He became more submissive in the shuttle box, but not in the home cage. Red initiated the most aggressive interactions, attacking Blue primarily and occasionally attacking White and Green. White's aggressive behavior was almost nil in both shuttle box and home cage. New SD hierarchy

As seen in Figure 15, the SD hierarchy was changed with Green's decrease in aggression (1354-357). White became a passive member of the hierarchy. Green showed decreased aggression, attacking Blue and less frequently, Red. Thus, almost by default, Red, third in the original SD hierarchy, became dominant over Blue, White and Green and initiated the most aggressive interactions $(98-710)$. The SD hierarchy was essentially reduced to interactions between Red, Blue and Green. Reaction to a fearful stimulus and the Kluver-Bucy syndrome

Within two days of his operation, Green approached and sniffed objects placed near his cage. Eight days after operation, Green escaped from the carrying cage and for several minutes proceeded to pick up objects on a table and sniff them. This behavior continued until Green was sacrificed six months later. Postoperatively, Green showed penile erections which lasted up to two minutes and which may have been indicative of hypersexuality. Although preoperatively all monkeys were observed to have erections, none of them were as prolonged as Green's postoperatively.

When Green was tested with the teddy bear (Table 2), Green crossed into the bear's compartment and sniffed the bear within 10 seconds after being placed in the opposite compartment. Green did 
not sniff the bear again, and crossed into the bear's compartment once more during the five-minute exploratory period. Preoperatively, Green had been tested twice with the bear and never crossed into the bear's compartment during the exploratory period. Postoperatively, when Green was shocked, he crossed into the bear's compartment and then immediately returned to the neutral compartment. Neither Red nor Blue crossed into the bear's compartment during the exploratory period, but did cross and immediately return to the neutral compartment on the first and fifth shocked trials, respectively. When White was tested with the bear, he immediately crossed into the bear's compartment, sniffed the bear, touched the bear and crossed back and forth between compartments seven times.

Green was tested for reaction to handling, and as in White's postoperative behavior, Green no longer violently avoided the approach of the glove. Green vocalized, bit the glove and struggled when held. White still struggled when held and Red and Blue's behavior remained the same as it was after White's lesion.

In summary, Green showed the following components of the Kluver-Bucy syndrome: sniffing behavior, visual agnosia, decrease in aggressive behavior and questionable hypersexuality.

\section{Discussion}

Green showed the same behavioral changes that White showed postoperatively. Green's aggressive behavior decreased, as did White's, more dramatically under negative than positive reinforcement. Recognizing that reinforcement was confounded with order of testing, there is some evidence for a differential effect of reinforcement on SD after temporal lobectomy. Postoperatively, 
White became less aggressive, more passive and no more submissive (was not attacked more). This pattern was also seen in Green's behavior, although Green was less passive than White. Green continued, until he was sacrificed, to initiate more aggressive interactions than White.

The relationship between a decrease in food-getting and a decrease in aggression after lesion was not consistent. After Green's lesion, he showed decreased aggression and food-getting in the shuttle box, but increased food-getting and decreased aggression in the home cage. After Green's lesion, White obtained the most food in the home cage and shuttle box, but decreased in aggression in both testing situations. Therefore, there is evidence that the decrease in aggression is independent of food-getting behavior.

At the end of testing under positive reinforcement, Green developed a tail infection. Before testing could resume, it was necessary to capture and apply medication to Green's tail once a day for two weeks. After two weeks of handing, Green was no more tame than at the beginning of testing.

Comparing size of lesions with behavioral changes, Green's lesion was similar to White's, in that both animals had bilateral sparing of the amygdala and bilateral destruction of medial and polar temporal cortex. The behavioral changes were also very similar. Both monkeys showed a partial decrease in aggression under positive reinforcement and almost complete disappearance of aggression under negative reinforcement. 
Part 3: Changes Following Red's Operation

Following Green's operation, Red initiated the most aggressive interactions and was the next monkey operated.

Positive reinforcement

Monkeys were individually tested for retention of the shuttle response. Only Red did not reach criterion and made $9 / 20$ correct responses. Red was tested three more days before reaching criterion. Throughout the retesting period, Red showed the following response pattern: he would make five to ten correct responses, curl up and stop responding for a few trials and then resume responding.

On the first trial of group testing in the shuttle box, Red did not compete for food and assumed a curled position. Except when escaping Blue's attacks, Red remained in this position throughout the testing sessions.

In the shuttle box (Figure 8), Red's aggressive score decreased from 256 to 0 , and he was more submissive (69-96), because of Blue's attacks. The scores in parentheses represent the scores after Green and Rec's lesion, respectively. Green continued to show less aggression $(222-31)$, continued to attack Blue and was never attacked. White was passive, showed minimal aggression and no submission, because Red no longer attacked White. Blue, the lowest monkey in the original SD hierarchy, attacked Red on the second trial of the first day of group testing and continued to dominate Red. Blue's aggressive score increased from 0 to 96 and he was less submissive (354-38), because Red ceased to attack him. Red did not cross the tunnel nor did he compete for food. As seen in Figures 9 and 10, the order for tunnel crossing and food-getting was White, Blue and Green. 
Home cage data is presented in Figure 11. During the first testing session in the home cage, Red showed catatonic-like behavior. He went to a corner of the cage and hung motionless on the side of the cage for the thirty-minute testing period. If another monkey approached Red, Red withdrew to a vacant corner of the cage and resumed his catatonic-like behavior. By the fifth and sixth session, Red was more active and less catatonic in the home cage and shuttle box. Red's aggressive behavior decreased $(43-0)$ and he was more submissive $(7-33)$, because of Blue's increased attacks. Blue was more aggressive (7-35) and still submissive $(102-67)$, as Green continued to attack Blue. Green's aggressive score remained at the same level (low 60's) as after his own lesion and he was never attacked. White continued to decrease in aggression $(31-5)$ and was less submissive (27-2), because he was no longer attacked by Red. As seen in Figure 10, White, Green and Blue received food about equally ( 80 times), just as after Green's lesion. Red's food-getting behavior dropped from 42 to 0 .

In sumary, Red's aggressive score in the shuttle box and home cage decreased to zero, and he showed no competition for food in either situation. Red's S score increased in both situations because of Blue's aggression. White's aggressive behavior almost disappeared and Green showed a continued decrease in aggressive activity. Blue, the remaining normal monkey, began to attack and dominate Red.

Negative reinforcement

Monkeys were individually retested on shuttle response and all 
reached criterion. Data for the shuttle box are shown in Figure 12. Red's aggressive score dramatically decreased (350-0) and he was frequently attacked by Blue. White and Green showed minimal aggressive activity and Green continued to attack Blue. Neither White nor Green was attacked. Blue's aggressive score markedly increased (0-100), reflecting Blue's attacks on Red. Blue was less submissive (364-36) as a result of Red's lack of aggression. The order for tunnel crossing (Figure 13) was Blue, Green, White and Ned. The order after Red's lesion was similar to that after White and Green's lesion except that Red had exchanged with White for last position.

In the home cage (Figure 14) there was very little activity and few aggressive-submissive interactions.

In summary, Red's aggressive score decreased to zero in the shuttle box and home cage, he did not compete for food and was last (preoperatively third) in tunnel crossing. White's aggression remained minimal and Green showed some aggression against Blue. Blue, the remaining unoperated monkey, attacked and dominated Red.

\section{New SD hierarchy}

As seen in Figure 15, the SD hierarchy was changed after Red's lesion. In order of aggression initiated, Blue was first (241), Green second (135), White was third and usually passive (18) and Red showed no aggressive behavior (0). In the SD hierarchy formed after Red's lesion, Blue attacked Red, and less frequently Green attacked Blue. 
Reaction to a fearful stimulus and the Kluver-Bucy syndrome

Postoperatively, Red rarely approached and sniffed objects, and did not sniff other monkeys as operated White and Green continued to do. Red showed possible hypersexuality in the form of prolonged erections (two minutes) and genital displays to the experimenter (ten times) during observation of the monkeys in the home cage.

When Red was tested with the teddy bear, Red crossed into the bear's compartment within twenty seconds after being placed in the opposite compartment. As seen in Table 2, Red crossed into the bear's compartment and returned to the neutral compartment 12 times during the five-minute exploratory period. Red sniffed the bear only once. When Red was shocked, he immediately crossed into the bear's compartment and then returned to the neutral compartment. Green was retested and did not cross into the bear's compartment during the exploratory period, but did cross and return to the neutral compartment when shocked. White continued to show the same exploratory and crossing behavior as when tested after his and Green's lesion. White crossed and returned to the neutral compartment twenty-six times, spent two minutes in the bear's compartment sitting next to bear, sniffed and touched the bear once. Blue crossed to the bear's compartment only when shocked.

Red was tested for reaction to handling, and like White and Green's postoperative reaction, Red showed no fear of the glove until he was grasped, at which time Red struggled and bit the glove. Blue could be handled and he struggled when held.

In summary, Red showed the following components of the Kluver- 
Bucy syndrome: negligible sniffing behavior, visual agnosia, complete absence of aggressive behavior and questionable hypersexuality.

\section{Discussion}

Red showed more extreme behavioral changes than White and Green had shown postoperatively. Unlike White and Green, who continued to initiate aggressive activity, Red showed no aggression in any of the four testing conditions. Red showed submissive behavior when attacked and chased by lower ranking Blue. An interesting sequence of aggressive behavior was noted. Frequently, when Green attacked Blue, Blue then attacked Red. Red ran into the sides of the testing compartment in attempts to avoid Blue's aggression. At no time did Red defend himself or fight when attacked by Blue.

Neither White nor Green showed the extreme catatonic-like behavior that Red displayed. The catatonic behavior was gone three weeks after the operation.

At the time of operation, Red weighed 2 pounds and 11 ounces. After surgery, Red's weight decreased over a period of three weeks to 2 pounds. To return Red to preoperative weight, Red was allowed to eat $\underline{a d} \underline{l b}$ four to eight hours daily in an individual cage, given extra fruit and sugar, and his cage was placed under a light while eating for it seemed that light stimulation made him more active. None of these measures increased Red's weight above 2 pounds. It appeared that Red began to eat normally when a food box was put on his cage, but after 10-15 minutes he stopped eating, although there was still food present. In comparison, White and 
Green (operated), and Blue (normal) continued to eat for another 30-40 minutes until all their food was gone. When Red was sacrificed almost five months later, he still weighed 2 pounds. None of the other monkeys showed any pre- or postoperative weight changes.

As discussed earlier, White, Green and Red were apparently of Colombian or Peruvian origin (Gothic type), while Blue (Roman type) was probably native to Brazil. MacLean (1964) found that Gothic monkeys made a genital display to a mirror while the Roman monkeys rarely did. According to MacLean, there were no differences in aggressive or courtship patterns of the two varieties. In the first group testing session after Red's lesion, and before Blue was operated, Blue displayed to Red five times. Although Blue had never displayed before, his genital display pattern was indistinguishable from that of the Gothic monkeys' pattern. The fact that Blue displayed to and attacked Red, after Red's operation, is evidence against the hypothesis that Blue was the lowest in the SD hierarchy because he was a different variety of squirrel monkey and, therefore, might have different aggressive-submissive patterns.

Red had almost a complete amygdalectomy and showed no aggression nor competition. In contrast, White and Green both showed aggression and both had portions of the amygdala spared bilaterally. Thus, it appears that unless the amygdala is completely removed, the monkey may continue to show aggressive behavior.

Red also had bilateral optic tract damage. Immediately after surgery, Red showed a transient visual guidance deficit in approaching and licking Sustagen from a syringe. Three weeks later, Red went 
directly to and picked up a single dextrose pellet, four feet away, that had fallen out of the food cup into the bottom of the shuttle box. It appears that the optic tract damage did not cause a serious visual deficit.

Red had a portion of right superior temporal and inferior frontal cortex with complete neural atrophy and gliosis. This was probably the result of interrupted blood supply to that area. It is not known how much this damage contributed to the behavioral changes, but unilateral lesions rarely, if ever, result in the gross emotional and behavioral changes seen in Red. There remains, of course, the possibility that the combination of damage, rather than the total amygdalectomy, was responsible for Red's marked changes.

When retested on the shuttle response with positive reinforcement, Red showed a deficit in performance. The deficit could have been the consequence of visual impairment as a result of optic tract damage or possibly another manifestation of the catatonic behavior seen in this subject. During retesting Red was observed to stop responding and assume a curled position. In any case, the deficit disappeared in four days.

Part 4: Changes Following Blue's Operation

After Red's operation, Blue, last in the original SD hierarchy, initiated the most aggressive behavior and was operated. Positive reinforcement

The monkeys were individually retested for retention of the shuttle response. White, Green and Red reached criterion on the first day. Blue did not cross in the first 14 trials, then made 
six correct responses $(6 / 20)$. During the first 14 trials, Blue sat on the empty food cup (his usual position), showed orientation to the buzzer, but did not cross through the tunnel to the baited food cup. The next day, Blue reached criterion.

During the first group testing session, Blue did not compete for food. When Green or White approached and sniffed Blue, Blue vocalized and often attacked the investigating monkey. At the end of positive reinforcement testing, Blue no longer showed any reaction when the other monkeys approached and sniffed him.

The shuttle box data is shown in Figure 8 . Blue's aggressive score decreased $(96-51)$ and he was attacked more frequently $(38-76)$ by Green. The scores in parentheses represent the scores after Red and Blue's lesion, respectively. Green showed more aggression (31-84) and more submission $(0-38)$, as Blue and White infrequently attacked Green. White showed minimal aggression and submission. Red displayed no aggression and was attacked less (96-26), as a result of Blue's decreased aggression. The order of tunnel crossing and food-getting was the same as it was after Red's lesion (Figures 9 and 10). White crossed most frequently and received the most food, Blue second, Green third and Red did not cross.

In the home cage (Figure 11), Blue was less aggressive (35-22) and less submissive $(67-1)$, as Green attacked Blue less frequently. Green's aggressive behavior dropped from 63 to 0 . Neither Red nor White showed any aggressive behavior and Green, Red and White showed little or no submissive behavior. Although White and Green showed no aggressive behavior, they competed with Blue for food (Figure 10). Blue competed for or received food 82 times, White 81 times, Green 38 and Red 0 times. 
In summary, Blue showed less aggression in the shuttle box and home cage while food-getting behavior decreased only slightly. Blue was attacked more in the shuttle box than in the home cage. Red was attacked less frequently by Blue. White's aggressive and submissive behavior was minimal. Green was aggressive in the shuttle box but not in the home cage. Thus, Green attacked Blue, Blue and White attacked Green and Blue attacked Red.

\section{Negative reinforcement}

Monkeys were individually retested for retention of the shuttle response and all reached criterion. In the shuttle box (Figure 12), Blue's aggressive score dropped from 100 to 0 . Blue's submissive score remained about the same $(36-31)$, as White and Green attacked Blue 10 and 21 times, respectively. White and Green showed little submissive behavior. Red showed no aggression and was attacked less $(97-6)$, because of Blue's lack of aggression. The order of crossing the tunnel after Blue's lesion (Figure 13) was Green, Blue, Red and White. Blue consistently had crossed the tunnel first since White's lesion and now crossed first (30) and second (36) equally. Red and White had again exchanged third and fourth positions.

In the home cage, aggressive and submissive behavior (Figure 14) was minimal for all monkeys. During the thirty-minute observation periods, the four monkeys either sat on the perch or searched for food on the bottom of the cage.

In summary, Blue's aggressive score in the shuttle box and home cage dropped to zero. Blue's submissive score remained low. Red was less submissive because of Blue's lack of aggression. There was little change in White and Green's aggressive or submissive scores. 


\section{New SD hierarchy}

As shown in Figure 15, the SD hierarchy changed after Blue's lesion. In order of frequency of aggressive responses, Green was first (121), Blue second (77), White third (54) and Red showed almost no aggressive behavior (8). Thus, the final SD hierarchy, after the four monkeys were lesioned, was characterized by little aggressive activity. The total number of aggressive interactions in the original hierarchy was 1848. After the four monkeys were lesioned, the total was 260 . This represents a reduction to oneseventh of the initial level of aggression. Reaction to a fearful stimulus and the Kluver-Bucy syndrome

Blue showed little approach and sniffing behavior and no hypersexuality.

When Blue was tested with the teddy bear, he did not cross into the bear's compartment during the five-minute exploratory period. He did cross into the bear's compartment when shocked, and avoided the bear, immediately returning to the neutral compartment. Blue was the only monkey after lesion who did not approach and sniff the bear during the five-minute exploratory period. As seen in Table 2, White and Green continued to cross into the bear's compartment during the five-minute exploratory period and each sniffed the bear once. Red did not cross into the bear's compartment during the exploratory period. When shocked, Red crossed into the bear's compartment, avoided the bear and immediately returned to the neutral compartment.

Blue showed the same reaction to being handled as he had preoperatively. Blue attempted to avoid being handled and struggled when held. White, Green and Red all continued to struggle when held. 
In summary, after lesion, Blue showed the following components of the Kluver-Bucy syndrome: some sniffing behavior, decrease in aggressive behavior, no visual agnosia nor hypersexuality.

\section{$\underline{\text { Discussion }}$}

Blue did not show the characteristic fearlessness, approach and sniffing behavior in response to the teddy bear that the other three operated animals showed. Blue did show some exploratory behavior, for he was observed to approach, grab and sniff the experimenter's glasses as the experimenter was engaged in watching the other monkeys.

Blue showed a greater decrease in aggression under negative than positive reinforcement. Of the four lesioned monkeys, three showed a differential decrease in aggression with a greater decrease in aggression under negative than positive reinforcement. Red showed no aggression at a11.

At the beginning of testing under positive reinforcement in the shuttle box, Blue oriented to the buzzer and frequently went to the empty food cup in his own compartment instead of crossing the tunnel to the baited food cup, or watched White cross and followed White. In the home cage, Blue oriented to the buzzer by looking around but did not move toward the food cup until White started toward the food cup. It appears as if the buzzer had lost its cue function and that Blue used White's movement toward the food cup as the CS. After Red's lesion, Blue (unoperated) had obtained food almost as many times as he crossed the tunnel. Postoperatively, Blue crossed the tunnel over 50 times but obtained food only 25 times. Blue usually did not move toward the tunnel 
until White started to cross, so that White had the advantage. As positive reinforcement testing continued, Blue resumed responding to the buzzer by movement toward the baited food cup.

During observation in the home cage after Blue's lesion, Red mounted White seven times. This was the only observation of mounting with erection and pelvic thrusts among the monkeys. Immediately after surgery, Red displayed to the experimenter and, after Blue's lesion, Red displayed twice to the experimenter. It is questionable whether Red's mounting and display behavior was indicative of hypersexuality, since hypersexuality is usually defined as indiscriminative sexual mounting.

After surgery, Blue, Green and White all competed for food in the home cage while Red never attempted to obtain food. If Red was alone at the food cup when the pellets were dispensed, Red immediately withdrew from the food cup area.

It is difficult to explain, on the basis of lesion differences between monkeys, the fact that Blue did not explore the teddy bear. Blue, Green and White had similar damage to medial and temporal polar cortex and hippocampus. Blue had less sparing of the amygdala. A behavioral explanation is possible. General level of activity may be closely related to the occurrence and frequency of approach behavior seen postoperatively. Of the four monkeys, Blue was the least active in all testing conditions.

Part 5: Comparison of Results from White, Green, Red and Blue's Operations

All monkeys initiated fewer aggressive interactions after their lesions (Figure 15). White, Green and Blue showed a greater decrease in aggressive behavior under negative than positive reinforcement, 
but order of testing was confounded with reinforcement. Red showed no aggression under any testing or reinforcement contingency. Across sequential lesions and testing (Figure 15) White and Green continued to decrease in aggressive activity. White declined from a norma1 level of 884 aggressive responses to 427 after his lesion, 106 after Green's lesion, 18 after Red's lesion and 54 after Blue's lesion. Green displayed this same pattern of continued decrease in aggression across lesions. White, Green and Blue, although they initiated fewer agnostic interactions after surgery, continued to initiate aggressive behavior throughout the course of the experiment. Thus, postoperatively, all monkeys except Red continued to initiate minimal aggressive activity.

The frequency of aggressive interactions was intensified by positive or negative reinforcement and restricted space. When the monkeys were observed in their home cage without experimental manipulation, the frequency of aggressive interactions was always less than that observed during testing under reinforcement. The frequency of aggressive behavior was also greater in the restricted space of the shuttle box than in the larger home cage.

The change in the SD hierarchy after the dominant monkey was operated was similar to the change seen earlier in normal monkeys, when the dominant monkey had been removed from the group and the remaining monkeys were tested for linearity. After White was lesioned, Green initiated the most aggressive behavior and dominated Red and Blue. Green had dominated Red and Blue in earlier testing when White had been removed from the group. After Green's lesion, Red initiated the most aggressive behavior and dominated Blue. 
Red had dominated Blue in earlier testing when White and Green were removed from the group. After Red's lesion, Blue showed aggressive behavior for the second time since testing had begun. Blue initiated the most aggressive interactions and dominated Red. The first time Blue showed aggressive behavior was in the earlier testing of normal monkeys, when White was removed from the group and Green, Red and Blue were tested together. Green continuously attacked Red, and as Red ran around the compartment trying to escape from Green's attacks, Blue would attack Red. Thus, the change in the SD hierarchy after lesion resembled the changes noted earlier when the normal monkeys were tested for linearity. In postoperative testing, after the dominant monkey was lesioned, the monkey next in the SD hierarchy became the dominant monkey.

There is evidence that after surgery, a formerly dominant animal was not only less aggressive but, if attacked, showed submissive behavior (monkey did not defend or fight). After Green's lesion, Red attacked Green and Green tried to escape from attacks without defending or fighting as he had done preoperatively. Also, after Green's lesion, Green and Red attacked White, who did not retaliate. After Red's lesion, Blue attacked Red and Red did not defend or fight, but tried to escape from attack. Thus, after anterior temporal lobectomy, three monkeys who showed less aggression also showed submissive behavior (escape instead of defense) if attacked.

There was some relationship, postoperatively, between amount of food received and order of crossing the tunnel with decrease in aggressive behavior. Across testing conditions, postoperatively, 
(1) each dominant monkey showed a decrease in aggression; (2) White, Green and Red decreased and Blue remained about the same in foodgetting in the shuttle box; (3) White, Green and Red decreased and Blue retained about the same position in tunnel crossing under positive reinforcement; (4) Red showed a decrease, Green an increase and White and Blue remained about the same on food-getting in the home cage; (5) White, Red and Blue showed a decrease and Green retained the same position in tunnel crossing under negative reinforcement.

Across operations, postoperatively, (1) White continued to decrease in aggressive behavior, increased in food-getting in the shuttle box, remained about the same in food-getting in the home cage, increased from second to first in order of crossing tunnel under positive reinforcement and decreased from first to last in order of tunnel crossing under negative reinforcement. (2) Green continued to decrease in aggressive behavior, decreased in foodgetting in the shuttle box and home cage, decreased in tunnel crossing behavior in the shuttle box under positive reinforcement and increased from second to first in order of tunnel crossing under negative reinforcement. (3) Red continued to show no aggression, no food-getting behavior in the shuttle box or home cage, no tunnel crossing in the shuttle box under positive reinforcement and increased from fourth to third in order of crossing the tunnel under negative reinforcement. (4) Blue was tested once after operation and thus no data across operations were available.

As seen in Table 2, all monkeys except Blue showed visual agnosia. Visual agnosia was defined as present if, after temporal 
lobectomy, a monkey crossed from the opposite compartment into the teddy bear's compartment during the five-minute exploratory period. White, Green and Red crossed into the bear's compartment and smelled the bear within twenty seconds after they were placed in the opposite compartment. Preoperatively, none of the monkeys crossed into the bear's compartment during the exploratory period even after two to five exposures to the bear. Postoperatively, after White, Green and Red's initial approach to and sniffing of the teddy bear, they usually avoided any further contact with the bear, and often responded as if they still feared the bear's presence. For example, when in the bear's compartment, the lesioned monkeys always walked around the bear, by keeping close to the far side of the compartment as they did preoperatively. They usually returned immediately to the neutral compartment after crossing into the bear's compartment. Thus, although postoperatively, White, Green and Red showed visual agnosia, it appeared as if they retained part of their preoperative avoidance reaction to the bear. Blue reacted to the bear in an identical manner both pre- and postoperatively. It is difficult to explain the fact that Blue did not explore the bear on the basis of lesion differences because White, Green and Blue's lesions were similar.

Preoperatively, when monkeys were approached with the glove they violently avoided it by running about the compartment and into its sides. Postoperatively, the monkeys showed little avoidance to presentation of the glove (visual agnosia), but when the experimenter touched or held the monkey, it struggled, vocalized and bit the glove. No pre- or postoperative difference was noted in 
Blue's reaction to the glove or to being handled. Preoperatively, Blue, in contrast to the other three monkeys, did not show violent avoidance of the glove. Pre- and postoperatively Blue could be handled and when held, struggled to escape.

Although, postoperatively, the monkeys were rarely observed to explore objects by putting them in their mouths, the four monkeys did approach, sniff and lick objects and other monkeys. The monkeys could be ranked according to the frequency of their sniffing other monkeys. After Blue's lesion, summing across all testing conditions, White sniffed other monkey's feet, anogenital area and tail, 132 times; Green sniffed other monkeys 51 times; Blue, three times, while Red was never observed to approach and sniff other monkeys.

After lobectomy, hypersexuality was either absent or questionable in the four monkeys. Postoperatively, White and Blue showed no hypersexuality, while Green and Red showed questionable hypersexuality. Green showed prolonged erections (up to two minutes). Red sexually displayed to the experimenter, and Red, with erection and pelvic thrusts, sexually mounted White. Preoperatively, no sexual mounting was observed, Red did not display to the experimenter, and although all monkeys showed frequent erections, none of the erections were as prolonged as those observed postoperatively. Thus, postoperatively, there was only suggestive evidence for hypersexuality.

Postoperatively, when tested for retention, all monkeys showed a transient deficit in performance on the shuttle response for positive reinforcement, but not for negative reinforcement. All 
monkeys reached criterion on retesting in two to four days with positive reinforcement. Red, who had almost a total amygdalectomy and bilateral optic tract damage, required four days to reach criterion. Thus, although the shuttle response was a simple and overlearned task, all monkeys showed a transient deficit in performance on the shuttle task with positive reinforcement.

$$
\text { Part 6: Discussion }
$$

The results of the present study on the squirrel monkey are in general agreement with the results reported on the rhesus monkey (Rosvold et al., 1954). Both dominant squirrel and rhesus monkeys show a decrease in SD after temporal lobectomy. In the Rosvold et al. study, one of the three operated monkeys did not decrease in dominance after surgery. This was attributed to either sparing of the basolateral nucleus or to the non-aggressive group environment to which the monkey was returned postoperatively. In the present study, postoperatively, all monkeys decreased in SD and one monkey showed no aggression. The operated squirrel monkey was passive and rarely attacked when retested with the group (except Red), in contrast to the rhesus study in which the operated monkey was aggressively attacked after surgery. Thus, for the squirrel monkey, the non-aggressive group environment was not a critical factor in the postoperative decrease in SD.

Although markedly below preoperative aggressive levels, the three monkeys in which some part of the amygdala remained, continued to initiate aggressive behavior. Red, with almost total amygdalectomy (also right inferior orbital and temporo-frontal cortical damage) showed neither aggressive nor competitive behavior. It 
appears that unless the amygdalectomy is complete, aggressive behavior may still be mediated by the remaining portion of the amygdala.

The monkey's behavior after lobectomy could best be described as passive and reactive rather than submissive. The lesioned monkeys showed most of their aggressive activity after being shocked, attacked, run into or prevented from getting food. It is suggested that incomplete amygdaloid lesions resulted in a heightening of the monkey's rage threshold while complete amygdalectomy almost totally abolished the rage response.

The three monkeys with some degree of amygdala spared (White, Green and Blue) showed a greater decrease in aggressive behavior under negative than under positive reinforcement testing. Because order of testing was confounded with reinforcement, the differential decrease in aggression could be due to either the differences in reinforcement or to some other process occurring during the recovery and testing period. The latter suggestion is based on the fact that the monkeys were tested on positive reinforcement two weeks after surgery and negative reinforcement six weeks after surgery. When the testing series began again, after the next monkey's lesion, the previously lesioned monkey now showed about the same low frequency of aggressive interactions under positive reinforcement that he had shown at the end of negative reinforcement on the previous testing. The fact that the lesioned monkey remained at the lower negative reinforcement level of aggression supports an explanation based on the time differences in order of testing to account for the differential decrease in aggression. 
It might also be suggested that the postoperative testing series was a "learning experience" for the lesioned monkey and that the experience of negative reinforcement testing more or less permanently altered the monkey'S SD behavior. Thus, in future testing, the monkey displayed the decreased aggression "learned" under negative reinforcement. The latter explanation receives some support from Weiskrantz (1956), who reported that a monkey treated gently and as a pet after amygdalectomy remained tame for several months. However, an amygdalectomized monkey treated indiscriminately, or as a normal laboratory animal, was grossly indistinguishable from a normal monkey during the six-week period. Thus, postoperative handling experience can markedly change an operated animal's behavior. It is possible that negative reinforcement, like handling, may have a dramatic effect on SD behavior.

The postoperative differential decrease in aggression is also confounded with the fact that under positive reinforcement the monkeys were food deprived and tested at 90 per cent body weight, while under negative reinforcement they were tested at normal body weight. The monkeys might be expected to be more active under food deprivation and initiate more aggressive interactions. However, the total number of aggressive responses the normal monkeys made in the shuttle box-positive reinforcement (624) versus shuttle boxnegative reinforcement (629) was about equal. Thus, there is little evidence for the hypothesis that the food deprivation increased activity, which then increased aggression. Whether level of food deprivation and order of testing or the different reinforcement contingencies accounted for the differences in aggression can only be determined by future experimentation. 
Amygdalectomized rhesus monkeys have been described as tame, placid, fearless (Walker et al., 1953; Orbach et al., 1960; Anand et al., 1959) in their reaction to man. Because the normal squirrel monkey did not show the marked avoidance and/or threatening pattern to humans that rhesus show, tameness was difficult to observe postoperatively in squirrel monkeys.

Three of the four monkeys did show visual agnosia. In the rhesus monkey, visual agnosia to a variety of feared and novel stimuli has been reported (Kluver \& Bucy, 1939, Anand et al., 1959; Masserman et al., 1958). Three squirrel monkeys showed little or no fear reaction to a previously feared teddy bear or to a glove used to catch monkeys. The agnosia appears to be somewhat specific to the visual system, for when the lesioned squirrel monkeys were grabbed or held, they reacted with appropriate escape behavior. Amygdalectomized rhesus monkeys who showed fearlessness and visual agnosia reacted with directed aggression when handled (Downer, 1962; Anand et al., 1959). Thus, temporal lobectomized squirrel and rhesus monkeys show reactions to visual istimuli which are different from their reactions to tactile and other stimuli involved in being handled.

It is known that damage to the infero-temporal cortex produces impairment on visual discrimination (Mishkin, 1954; Brown et al., 1963). Mishkin (1954) suggested that the discrimination impairment seen in temporal lobectomy was due to involvement of the ventral cortex of the temporal lobe. He also reported that damage to the hippocampus or amygdala and hippocampus resulted in little or no impairment on visual discrimination. Wood (1958) reported that he 
did not observe visual agnosia in cats with small amygdaloid lesions. Thus, there is some evidence that the visual agnosia component of the Kluver-Bucy syndrome may result from infero-temporal and/or extensive amygdaloid damage.

As observed by others (Kluver and Bucy, 1939; Walker et al., 1953; Terzian and Ore, 1955), some of the behavioral changes which resulted from temporal lobectomy were long lasting. In the present study, White postoperatively showed sniffing behavior, visual agnosia and decreased aggression until sacrificed almost a year later. Postoperatively, all the other monkeys showed decreased aggression, while visual agnosia and sniffing behavior decreased somewhat with time.

Postoperatively, all monkeys showed a transient (2-4 day) deficit on retention of the shuttle response under positive reinforcement. Monkeys with amygdaloid-hippocampal damage have been reported to show deficits on simple visual and somesthetic tasks as well as on complex delayed matching, delayed alternation and compound stimuli discrimination tasks (Stepien et al., 1960; Drachman \& Ommaya, 1964; Orbach et al., 1960). Most of the deficits reported following hippocampal-amygdaloid damage are on complex tasks. Because the shuttle task was a simple and preoperatively overtrained response, no retention deficit would necessarily be predicted. A transient deficit was observed and it is possible that this deficit in retention resulted from bilateral hippocampal involvement.

The ablation of the amygdaloid complex in many different species has resulted in similar behavioral changes. In the present study, the postoperative behavior of the squirrel monkey was similar to 
that of the rat (Bunnel1, 1966), dog (Fuller et al., 1957), rhesus monkey (Rosvold et al., 1954) and human (Terzian \& Ore, 1955). Thus, there is evidence of a similar brain structure influencing the same behavioral functions across several species.

There is as yet no unitary concept to incorporate and explain the results of amygdalectomy or amygdaloid stimulation on socialemotional and learning behavior. The amygdala has been reported to be involved in response perseveration (Mahut \& Cordeau, 1963), determination of the discriminative and reinforcing properties of stimuli (Schwartzbaum \& Pribram, 1960), habituation or satiation which way or may not be specific to food (Schwartzbaum, 1961), adaptation to sustained novelty properties of stimuli (Schwartzbaum, 1964), emotional reaction to aversive or frustrating conditions (Schwartzbaum et al., 1964), active suppression of motivated approach behavior (Goddard, 1964), correlation of past experience and present sensation with the appropriate motivational responses (Gloor \& Ferndel, 1963), the facilitation of hypothalamic activity (King, 1958; King \& Meyer, 1958) and threshold level for social stimuli (Bunnel1, 1966).

In agreement with Bunnell's study, the present experiment interprets the function of the amygdala as modifying the threshold level, and therefore the response level, to social and environmental stimuli. This interpretation is based on the observation that, although postoperatively the monkeys showed little or no aggression, they could respond with directed rage or aggression when aroused by shock, attack, competition, or handling. Thus, it appears that the social and environmental stimuli to which the animal responded 
with aggression preoperatively, would no longer elicit aggression or rage postoperatively, unless the operated animal was primed by alerting stimuli (shock, attack, etc.). In a simplified schema, if the amygdala is removed, the threshold for social stimuli is permanent1y raised (fearlessness, tameness, lack of aggression, visual agnosia) and if the amygdala is stimulated, the threshold is reversibly lowered (attention, fear, rage).

The present data might also be interpreted in terms of changes in discriminative and reinforcing properties of stimuli (Schwartzbaum \& Pribram, 1960). From this interpretation, it would follow that an operated animal is less aggressive because he can no longer evaluate the reinforcing properties of stimuli or discriminate the social cues that preoperatively elicited aggression. The reason that the first interpretation--change in threshold and response level--is preferred to Schwartzbaum's, is that the latter has difficulty explaining the occurrence of discriminative aggressive responses that an operated monkey can make when pre-aroused or alerted.

The amygdala could possibly modify the threshold and response level to social stimuli through changes in endocrine levels and/or interaction with other brain structures. Monkeys with. subtotal bilateral amygdalectomy show a strikingly diminished and delayed plasma 17-hydroxycorticosteriod response to a conditioned emotional stimulus, while stimulation of the amygdala produces an elevation in 17-hydroxycorticosteriod level (Mason, 1959). Castration, either before or at the time of amygdala injury, prevents development of hypersexuality which normally follows these lesions 
(Schreiner \& Kling, 1953). Thus, there is evidence for amygdalaendocrine interaction in emotional behavior.

The behavioral changes resulting from amygdaloid lesions have been further altered by making additional brain lesions. Placid amygdalectomized cats became vicious after hypothalamic lesions (Schreiner \& Kling, 1953; Kling et al., 1958) and explosive, septal-lesioned rats were tamed by amygdaloid lesions (King, 1958; King \& Meyer, 1958). Therefore, different changes in behavior result from a combination of lesions.

On the basis of the present and above studies, it is suggested that the amygdala modifies the threshold level, and therefore the response level, to social stimuli. It is further suggested that physiological (brain and endocrine) mechanisms for modification of this threshold do exist. 
TABLE 2

The number of spontaneous crossings into the teddy bear's compartment during the five-minute exploratory period-a test for visual agnosia

\begin{tabular}{lccccc}
\hline & Normal & $\begin{array}{c}\text { White } \\
\text { Operated }\end{array}$ & $\begin{array}{c}\text { Green } \\
\text { Operated }\end{array}$ & $\begin{array}{c}\text { Red } \\
\text { Operated }\end{array}$ & $\begin{array}{c}\text { Blue } \\
\text { Operated }\end{array}$ \\
\hline White & 0 & 19 & 7 & 26 & 19 \\
Green & 0 & 0 & 2 & 0 & 16 \\
Red & 0 & 0 & 0 & 12 & 0 \\
Blue & 0 & 0 & 0 & 0 & 0 \\
\hline
\end{tabular}


SQUIRREL MONKEYS

SHUTTLE BOX-PR
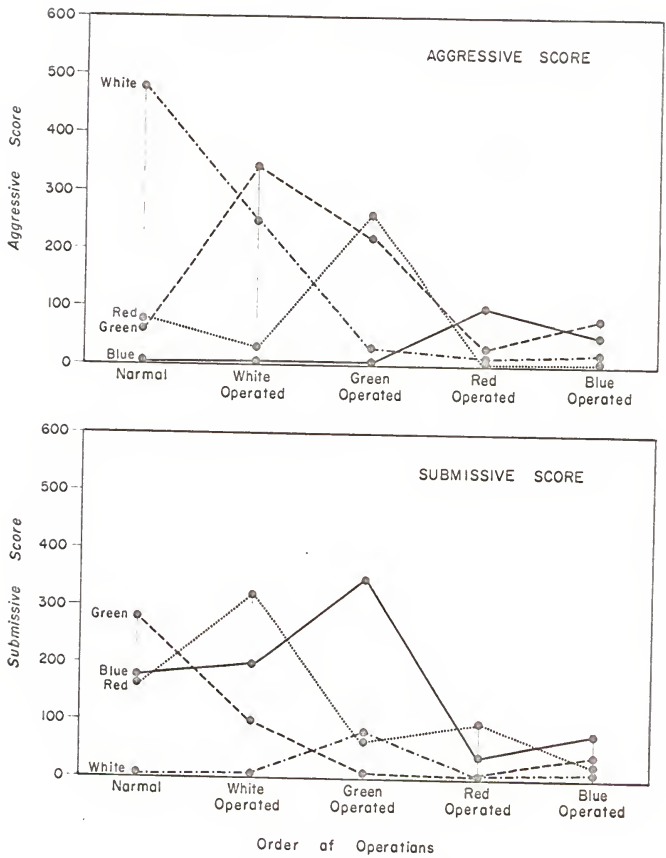

Figure 8. Aggressive and submissive scores for the normal monkeys and after the successively dominant monkey--White, Green, Red, and slue...was given a bilateral temporal lobectomy. Monkeys tested in shuttle box with positive reinforcement (PR). 


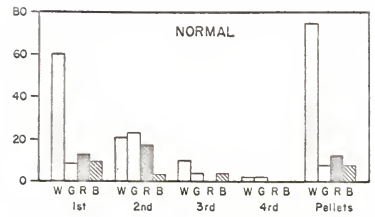

\section{SQUIRREL MONKEYS \\ SHUTTLE BOX -PR}
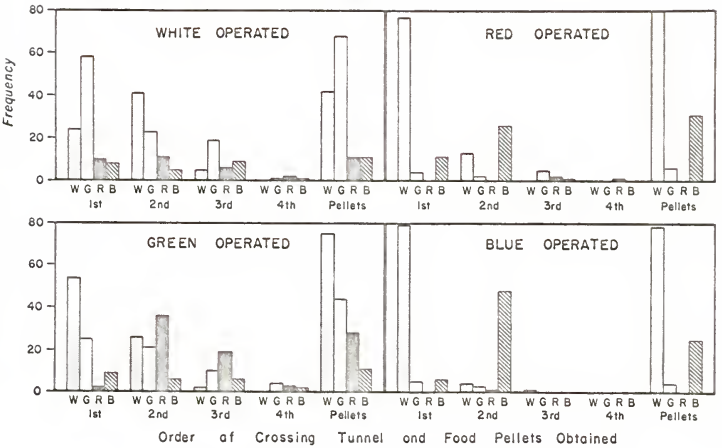

Figure 9. The order of crossing tunnal and food paliets obtained in the shuttle box, undar positive reintercement $(P R)$ lor the normal monkeys and after the successively dominant monky-- White, Green, Red, ant Blue-was given a bliateral temporal lobectomy. On the absissa, letters indicate monkeys and numbers $115 \mathrm{st}$. 2nd, 3rd, and $4(\mathrm{nl})$ indicate th
crossing orde?. Frequency of crossing is plotted on the ordinate. 


\section{SQUIRREL MONKEYS}

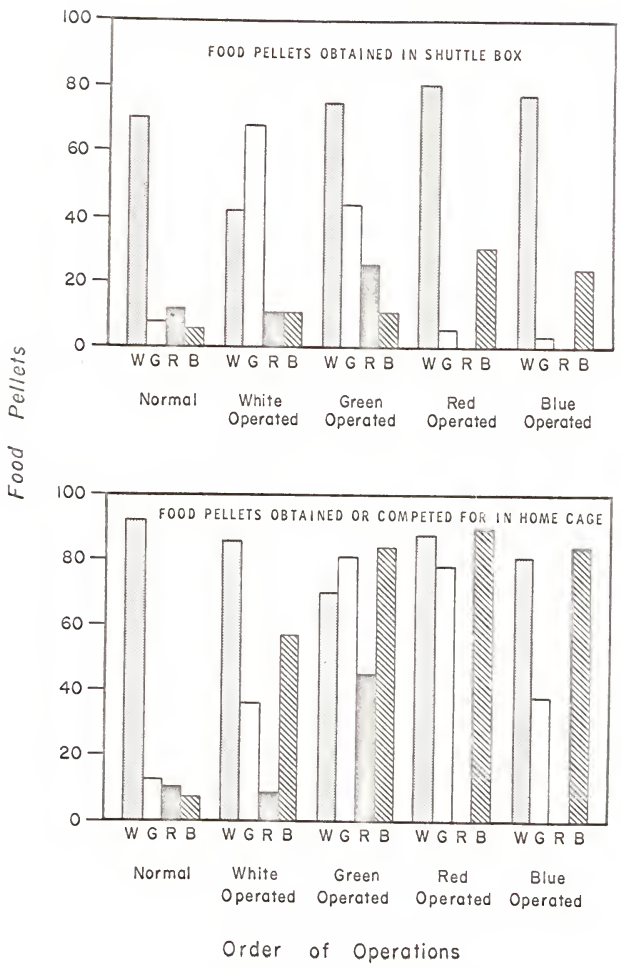

Figure 10. Food pellets obtained in snuttle box and food pellets obtained or competed for in home cage by normal monkeys and after the successively dominant monkey--White, Green, Red. and Blue--was given a bilateral teriporal lobectomy. 


\section{SQUIRREL MONKEYS \\ HOME CAGE-PR}
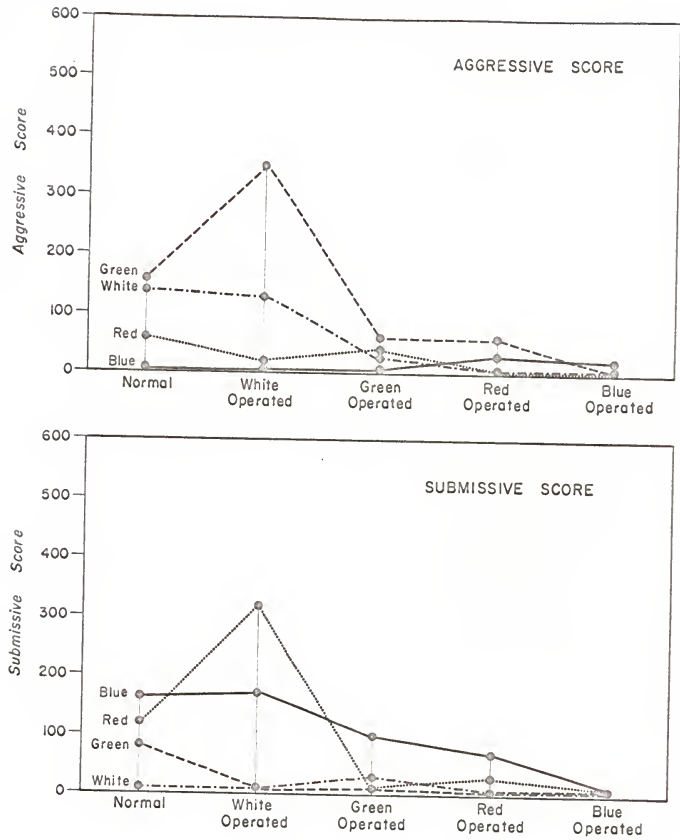

Order of Operatians

Figure 11. Aggressive and submissive scores for the normal monkeys and after the successively dominant monkey---White, Green, Red, and Blue--was given a bilateral. amporal lobectomy. Monkeys tested in the nome cage with positive reinforcement ( $P R$ ). 

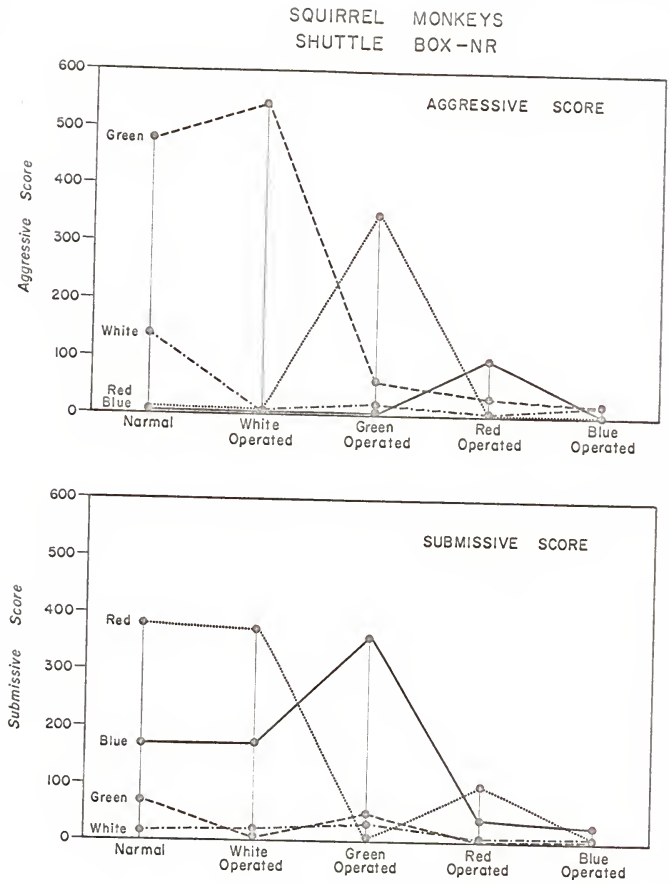

Order of Operations

Figure 12. Aggressive and submissive scores for the normal monkeys and after the successively dominant monkey---White, Green, Red, and Blue--was given a bllateral temporal lobectomy. Monkeys tested in shuttle box with negative reinforcement (NR). 


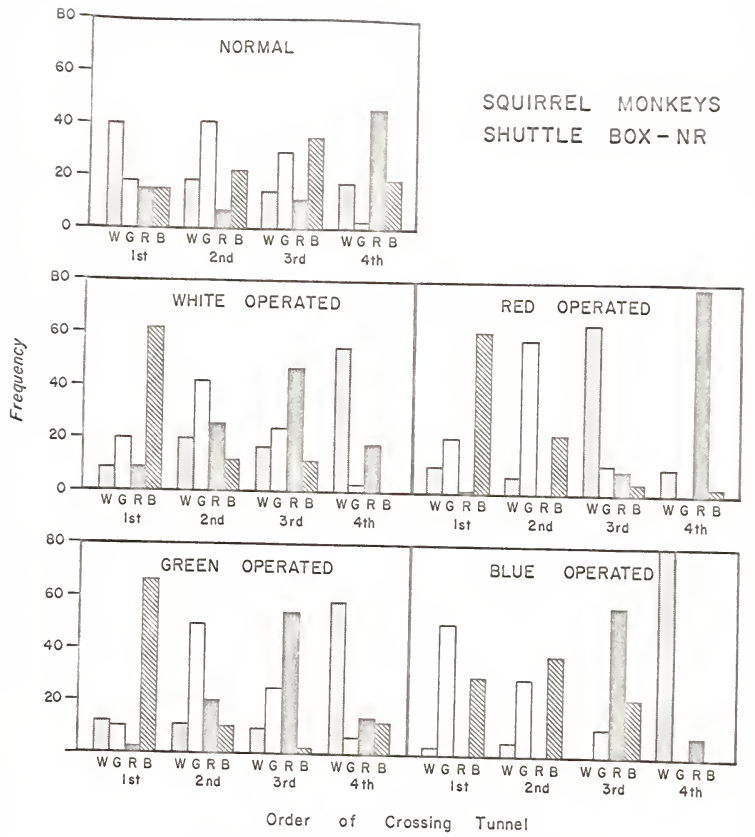

Figure 13. The order of crossing tunnel in the shuttlo box under negative reinlorcemen: (NR) for the normal monkeys and after the successively dominant monkey-..White, Green, Red and slue-.. was given a bilateral temporal lobectomy. On the absissa letter indicate monkeys and numbers (1st, 2nd, 3 rd, and $4 \mathrm{th}$ ) indicate the crossing order. Frequency or crossing is plotted on the ordinate. 


\section{SQUIRREL MONKEYS \\ HOME CAGE}
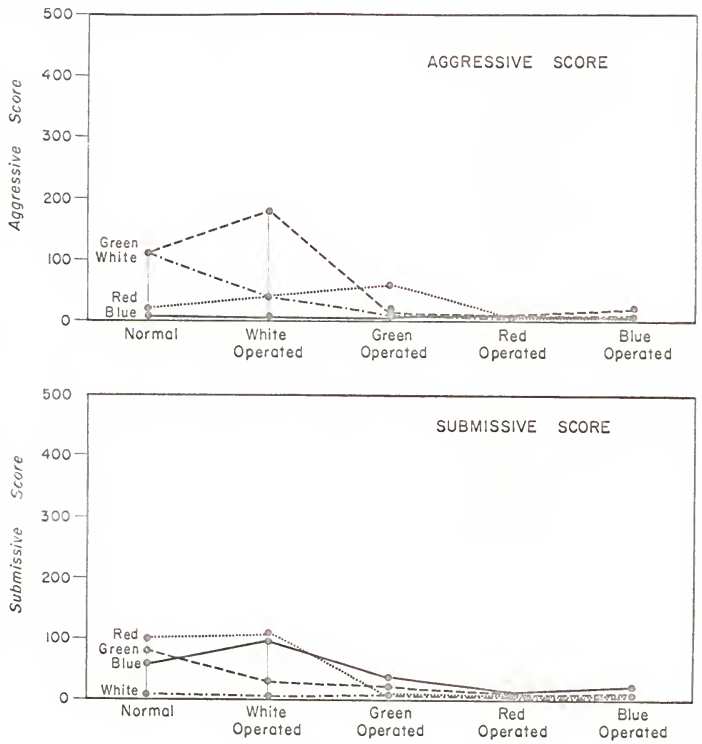

Order of Operotions

Figure 14. Aggressive and submissive scores for the normal monkeys and after the successively dominant monkey---White, Green, Red, and Blue-...was given a bilateral temporal lobectomy. Monkeys tested in home cage with no experimental manipulation. 
SQUIRREL MONKEYS
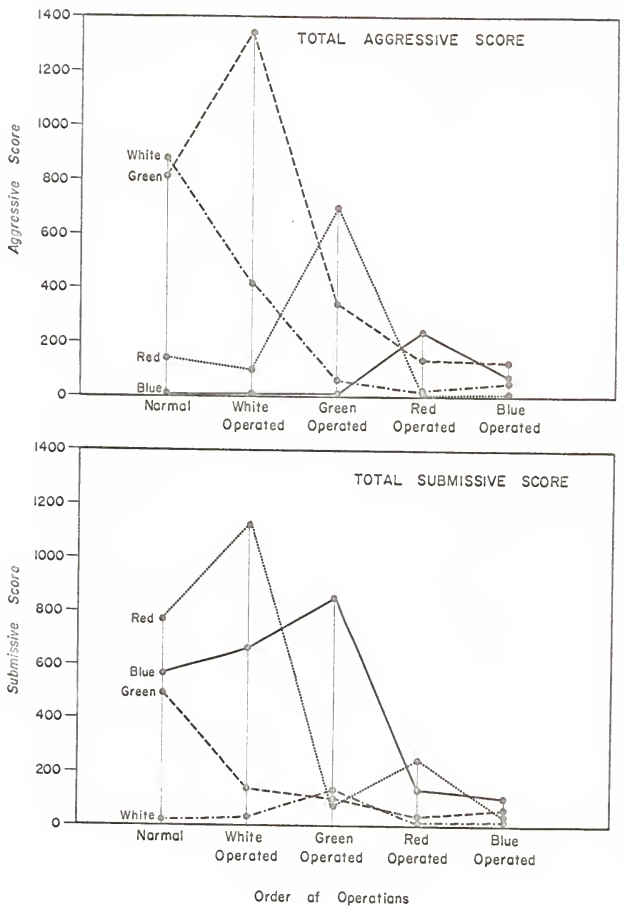

Flgure 15. Total aggressive and submissive scores, across all testing conditions, for the normal monkeys and after the successivety dominant

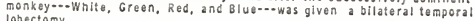
lobectomy. 


\section{EXPERIMENT III}

SD HIERARCHY IN THE CEBUS MONKEY AND COMPARISON OF SQUIRREL AND CEBUS MONKEY FINDINGS

In previous reports (Ploog et al., 1963; Bernstein, 1964, 1965) the social organization of the squirrel and cebus monkey has been evaluated, but not in a single study which provided identical experimental conditions. Perhaps the most profitable way to determine similarities and differences due to species variation is to hold testing experience constant. Data on the squirrel monkey's social dominance hierarchy has been reported in Experiment I, and is compared here with cebus monkey results collected under the same experimental conditions.

It is hypothesized here that the pattern of social organization and SD occur with varying degrees of hierarchial rigidity depending on the test conditions. In this context, the SD hierarchy should become more pronounced and rigid with increased and sustained intra-group competition. Therefore, the SD hierarchies reported in the present experiments for the squirrel and cebus monkey were compared with SD structures observed under less competitive situations (Ploog et al., 1963; Bernstein, 1965).

Part 1: SD Hierarchy in the Cebus Monkey

Method

\section{$\underline{\text { Subjects }}$}

Four male cebus monkeys, identified by plastic color-coded 
collars (red, green, blue and white) were used. They weighed between 3 and 4 pounds. The monkeys were judged to be subadult from the $\mathrm{X}$-rays which showed that none of the epiphyses were completely closed and that dentation was complete in only one monkey (Red). Two months before the initiation of testing, and throughout testing, the monkeys were housed as a group in a cage $2 \frac{1}{2} \times 3 \times 3$ feet. During testing for positive reinforcement (PR) monkeys were maintained at 80-90 per cent body weight and fed separately. During negative reinforcement (NR) monkeys were fed together ad lib.

\section{$\underline{\text { Procedure }}$}

The apparatus, procedure, scoring and order of testing were identical to those described in Experiment I.

\section{$\underline{\text { Results }}$}

\section{Positive reinforcement (PR)}

The data in Figure 16 represent combined shuttle box and home cage behavior for each reinforcement contingency. The data were combined because the difference between these two test conditions was in the frequency, not in the direction, of aggressive interaction. As shown in Figure 16, the social hierarchy of the cebus monkeys under positive reinforcement was not completely linear. Red was the dominant monkey, followed in dominance by Green, while the ranking of Blue and White was unclear with respect to each other. Red crossed the tunnel first most frequently and Green crossed second most frequently. Blue and White interchanged positions three and four, usually neither crossing nor competing with Red and Green for food. In spite of Green's persistence in 
following Red through the tunnel, Green received pellets only twice while Red received pellets 90 times. White received one pellet and Blue obtained none.

In summary, Red dominated the other monkeys, received the most food pellets and crossed the tunnel first most frequently. Green, second in the SD hierarchy, dominated Blue and White. Blue and White's third and fourth positions in the hierarchy were reversible, since neither attacked the other. Negative reinforcement (NR)

As shown in Figure 16, the SD hierarchy appears linear under negative reinforcement, but the frequency of aggressive responses between Green, Blue and White was too low to assert that this was an unqualified linear social structure. Red was the dominant monkey and there was partial evidence for ordering Green, Blue and White in decreasing SD. There was little correlation between SD position and order of crossing tunnel to avoid or escape shock. Blue crossed first most frequently, Red crossed second most frequently, Green third and White last. A comparison of the frequency of aggressive interactions between PR and NR indicates a 50 per cent decrease in aggressive behavior under negative reinforcement.

Across reinforcement contingencies, grooming activity remained about the same with 77 interactions in the shuttle box-PR and 73 for shuttle box-NR. Red, the dominant monkey, initiated 134 of the 150 grooming interactions.

Thus, the social structure observed under NR was similar to that with PR. In both testing situations Red was dominant, Green 
appeared second in dominance, although Green, Blue and White's exact SD positions were inconclusive from the data.

\section{Discussion}

The appearance in the present study of a dominant cebus monkey and a probable SD hierarchy is in contrast to the results of Bernstein's study (1965). He did not find a "dominant male" based on priority for incentives, nor did he find evidence for a social status hierarchy. He did observe a cebus male who showed many significant features associated with the role of a dominant male macaque, but dominance seemed unimportant in the group. This discrepancy in findings way be due to differences in testing conditions. In the present study, the probability of physical interaction and aggressive behavior was maximized by limiting space to two $2 \times 2 \times 2$ feet experimental compartments and a larger home cage. Intra-group competition was sustained throughout the testing sessions by competition for food or shock avoidance, which probably generalized to the aggressive interactions between food or shock trials. In comparison, Bernstein (1965) observed 12 cebus monkeys (three males and six females were mature; one female and two males were immature) in two large outdoor runs, the smallest of which was $24 \times 48 \times 8$ feet high. The monkeys were allowed to feed ad lib. Both the size of the enclosure and the ad $1 \mathrm{ib}$ feeding would minimize the probability of aggression occurring. At no time were the monkeys in Bernstein's report surgically or behaviorally manipulated.

A possible indication of the importance of space in competitive behavior is the fact that Bernstein observed primarily 
aggressive threats and vocalizations, rarely actual combat. In the present study, the primary occurrences were of combat, biting and attacking, while threat behavior was rarely observed. Therefore, by limiting space and sustaining intra-group competition, a SD hierarchy may emerge which is obscured under less competitive conditions.

\section{Part 2: Comparative Data}

\section{$\underline{\text { Results }}$}

The primary difference between the SD hierarchies of squirrel monkeys (SM) and cebus monkeys (CM) is shown in Figure 16 and 17. The SM have a clearly defined linear SD hierarchy, while the CM social hierarchy comprises a dominant male and questionable SD hierarchy for the remaining monkeys. As seen in Figure 17, there was a substantial difference between total number of aggressive responses across all testing conditions, with 1859 responses for SM and 890 for CM. Thus, for the same time period, there were twice as many aggressive interactions for the SM compared with the CM.

The SM never engaged in the interanimal grooming which was frequently observed in CM. Furthermore, the CM never showed the genital display posture which was characteristic of the SD pattern of the SM. Both species showed intermittent "playful" behavior, which, except for intensity, was similar to aggressive behavior. In summary, the SD hierarchies for these two New World species, observed under the same testing conditions, was similar in that both groups have a dominant "boss" monkey, but different in the degree of rigidity of the SD structure. 


\section{COMPARISON DATA}
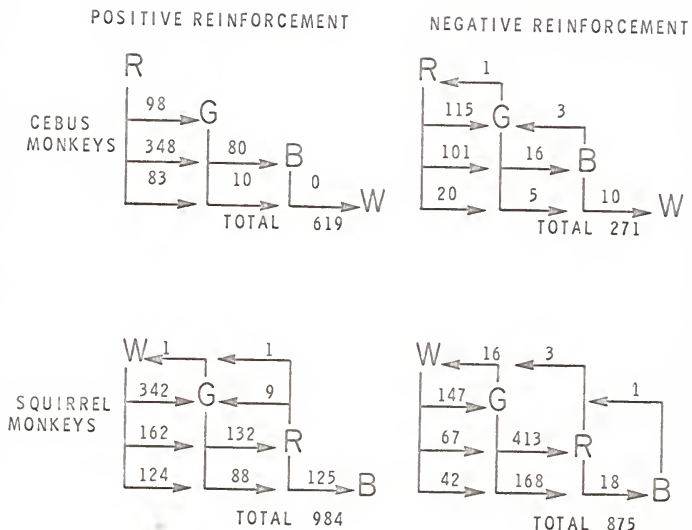

TOTAL 875

Figure 16. Frequency of aggressive responses (pushing, pulling, biting, attacking, chasing, mounting, and genital displayl recorded during the intertrial interval. Data combined for shuttle box and home cage. The letter indicates the monkey, the arrow indicates the direction of aggression and accompanying number indicates frequency of aggressive responses. 


\section{COMPARISON DATA}

CEBUS MONKEYS

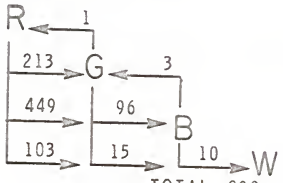

TOTAL 890
SQUIRREL MONKEYS

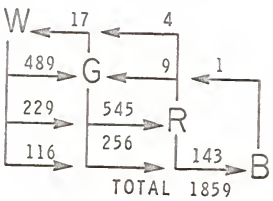

Figure 17. Frequency of aggressive responses across all testing conditions. The letter indicates the monkey, the arrow indicates the the direction of aggression and accompanying number indicates the frequency of aggressive responses. 


\section{Discussion}

The fact that the SM were adults and the CM were sub-adults may account for the finding of an incomplete linearity in the CM and almost complete linearity in the SM hierarchy. The dominant $C M$, Red, was also the heaviest monkey and only monkey to show complete dentation. The other three monkeys had incomplete dentation and weighed less than Red. All monkeys were ranked about equal in degree of epiphyseal closure and all were judged to be sub-adults. It is known that, in the rhesus monkey, dominance hierarchies of sub-adults are less linear than for adults (Kaufman, 1966). Therefore, in the present study, the difference in the degree of linearity between the SM and CM may be the result of differences in physical development, rather than of an essential behavioral difference between the species.

The fact that SM compared with CM showed twice as many aggressive interactions for the same amount of testing suggests that $\mathrm{CM}$ probably use less aggressive interactions to maintain their SD hierarchy. A second possibility is that adult monkeys show more aggressive interactions than sub-adults, and therefore SM would be predicted to show more aggressive responses than CM.

Results from both species indicate that the social hierarchy determined by priority for incentives is usually, but not always, correlated with the social dominance position as defined by aggressive interactions.

Comparing different testing procedures, the kind of social structure observed appears to be contingent upon the degree of competition in the testing situation. As discussed above, the 
differences between the present results and Bernstein's for the CM could be explained by the more competitive testing situations used by the former. For the SM, the degree of competition appears equally important. Ploog et al. (1963) analyzed the social behavior of six squirrel monkeys (three males and one female were mature; one male and one female were immature). The six monkeys were housed in a group cage which was only slightly larger than the home cage used in the present SM study. They observed the monkeys with and without experimental manipulation. The experimental manipulation consisted of four different 10-day periods, during which monkeys were given 10 food pellets daily in their home cage. At the time of testing, the animals had been deprived of food for 24 hours. The number of food pellets the monkeys received and/or "stole" were recorded. They found that the dominant monkey and the monkey second in dominance obtained about an equal number of pellets--130/400 pellets and 150/400 pellets, respectively. A monkey's dominance ranking was determined primarily from the occurrence and frequency of genital displays which dominant monkeys made to submissive animals. They did not report aggressive encounters between food trials. In comparison, squirrel monkeys in Experiment I of the present study were maintained at 90 per cent body weight during positive reinforcement testing. Under this condition, the dominant monkey (defined by frequency and direction of aggressive encounters) received $164 / 180$ pellets while the monkey second in dominance obtained $20 / 180$ pellets. In contrast to Ploog et al., these results show a large discrepancy between the pellets obtained by the first and second ranking dominant monkeys tested at 
90 per cent body weight. Thus, degree of competition, whether manipulated by space limitation, incentives, or deprivation, is a critical variable in research on social organization.

It is interesting to speculate whether evolutionary differences in cortical fissuration among the squirrel, cebus and rhesus monkey is related to the behavioral phenomenon of SD. Regarding fissuration, the brains of these species can be ranked from smooth brained to highly convoluted: SM, CM and rhesus monkeys. On the performance of complex behavioral tasks, the monkeys can be ranked in order of increasing proficiency: SM, CM and rhesus monkeys (Warren, 1965). With respect to the frequency and complexity of SD communication, there is some evidence from the present investigation and other studies for ranking the monkeys in increasing frequency and complexity: SM, $\mathrm{CM}$ and rhesus monkeys. The SM appear to show few dominant or submissive gestures (genital display). The CM show more dominant and submissive gestures (open mouth threat, submissive teeth chattering, vocalizations and mounting), as well as interanimal grooming. The rhesus monkeys show all the SD gestures seen in CM plus additional SD gestures. Thus, in a general way, there appears to be a relationship between increasing brain fissuration and increasing frequency and complexity of the SD communications observed in the social structure. 
SUMMARY

This study investigated three problems: (1) the measurement of the social dominance (SD) hierarchy in the squirrel monkey, (2) the effects of anterior temporal lobectomy on the SD behavior of the squirrel monkey and (3) the evaluation and comparison of the cebus monkey's SD hierarchy with that of the squirrel monkey. Regarding the first problem, behavioral tests were developed to assess the SD hierarchy of the squirrel monkey and later, the cebus monkey. The tests consisted of observing four monkeys over a period of seven months (1) in a shuttle box under positive reinforcement, (2) in their home cage under positive reinforcement, (3) in a shuttle box under negative reinforcement and (4) in their home cage with no experimental manipulation. In the first three of these tests, competitive trials for food or shock avoidance resulted in aggressive social interactions, which were expressed and recorded during the intertrial interval. The frequency and direction of the aggressive interactions were used to assess the position of an animal in the SD hierarchy.

The results from these four testing conditions indicated that the male, adult squirrel monkey has a linear SD hierarchy which is stable across reinforcement contingencies.

After the SD hierarchy of the squirrel monkey was assessed, the effect of a bilateral temporal lobectomy on SD behavior was 
studied during a period of one year. The dominant monkey of the previously tested group was operated and the group retested, using the behavioral tests developed earlier. After the dominant animal was operated, the monkey immediately below him in the original SD hierarchy became the dominant animal, was operated next and the group was retested. In this manner, the four successively dominant monkeys were operated and retested. In addition, monkeys were tested pre- and postoperatively for their reaction to a fearful stimulus and to being handled.

The effects of a temporal lobectomy on the dominant squirrel monkey's behavior can be summarized as follows: postoperatively, (1) each dominant monkey initiated fewer aggressive interactions and was less dominant, (2) three of the monkeys showed a greater decrease in aggressive responses when tested under negative reinforcement than when tested with positive reinforcement, (3) one monkey ceased to show aggression under any testing condition, (4) all monkeys could generally be described as passive or reactive rather than submissive, (5) three of the monkeys approached and sniffed a feared object (visual agnosia) that had been avoided preoperatively, (6) none of the monkeys showed any change in aggressive reactions to handling, although there was less display of fear to the glove used in handling, (7) there was little or no evidence of hypersexuality and (8) the behavioral changes were long lasting--up to the time of sacrifice one year later.

There was a positive correlation between amount of amygdala removed and decrease in aggression. One monkey with almost complete amygdalectomy showed no aggression. The other three monkeys 
had partial sparing of the amygdala and showed minimal aggression throughout testing.

The third problem evaluated and compared the SD hierarchy of the cebus monkey with that of the squirrel monkey. The cebus monkeys (four sub-adult males) were observed to have a dominant "boss" monkey and an incomplete linear SD hierarchy. In contrast, the squirrel monkeys were observed to have a complete linear SD hierarchy. 


\section{CONCLUSIONS}

The following conclusions were drawn from the study:

1. The SD structure can be observed with an increasing degree of hierarchial rigidity as the intra-group level of competition is raised.

2. Adult male squirrel monkeys have a linear SD hierarchy which, except for paucity of SD gestures, is similar to the social structure reported for adult male rhesus monkeys (Kaufman, 1966).

3. The squirrel monkey's SD hierarchy is stable across reinforcement contingencies, as reported for the rhesus monkey (Hamilton, 1960).

4. As in the squirre 1 and rhesus monkey's social structure, the cebus monkey's social organization has a dominant "boss" monkey, but unlike the other monkeys' SD hierarchies, the cebus monkey's is not completely linear.

5. There is a relationship between increasing cortical fissuration and increasing frequency and complexity of the SD communication observed in the social structure of the squirrel and cebus monkey and in reports on the rhesus monkey (A1tman, 1962).

6. The effects of a tempora1 lobectomy on the dominant squirrel monkey are similar to those reported for the dominant rhesus monkey (Rosvold et a1., 1954). Postoperatively, dominant monkeys of both species decrease in dominance and aggression. 
7. There is some evidence that after temporal lobectomy the dominant squirrel monkey shows a greater decrease in aggression when tested under negative reinforcement than when tested with positive reinforcement.

8. A temporal lobectomy results in similar changes across many different species (Rosvold et al., 1954; Terzian \& Ore, 1955; Fuller et al., 1957; Bunnel, 1966). This suggests that a similar brain region in different species modulates the same behavioral functions.

9. The amygdala's function is interpreted as modifying, either by endocrine changes (Mason, 1959; Schreiner \& Kling, 1953) and/or interaction with other brain structures (Schreiner \& Kling, 1953; King, 1958; King \& Meyer, 1958), the threshold level and, therefore, the response level to social and environmental stimuli.

10. Finally, infrahuman primate social behavior can be reliably assessed and behaviorally and surgically manipulated. For this reason, the study of infrahuman primate social behavior is a useful tool in the identification of the neurophysiological correlates of social behavior. 


\section{BIBLIOGRAPHY}

Adey, W. R., Buchwald, N. A., \& Lindsley, D. B. Amygdaloid, pallidal and peripheral influences on mesencephalic unit firing patterns with reference to mechanisms of tremor. EEG clin. Neuro-physiol., 1960, 12, 21-40.

Akert, K., Gruesen, R. A., Woolsey, C. N., \& Meyer, D. R. KluverBucy syndrome in monkeys with neocortical ablations of temporal lobe. Brain, 1961, 84, 480-498.

Altman, S. A. A ficld study of the sociobiology of rhesus monkeys, Macaca mulatta. Ann. N. Y. Acad. Sci., 1962, 102, 338-435.

Anand, B. K., Chhina, G. S., \& Dua, S. Effects of lesions in the limbic system on the affective behavior and visceral responses in the monkeys and cats. Indian J.med. Res., 1959, 47, 51-58.

Bagshaw, M. M., \& Pribram, K. H. Effect of amygdalectomy on transfer of training in monkeys. J. comp. physiol. Psychol., 1965, 59, 118-121.

Bard, P., \& Mountcastle, V. B. Some forebrain mechanisms involved in expression of rage with special reference to suppression of angry behavior. Res. Publ. Ass. Nerv. Ment. Dis., 1947, 27, 362-404.

Bernstein, I. S. A comparison of New and 0ld World monkey social organizations and behavior. Amer. J. phys. Anthrop., 1964, 22, 233-238.

Bernstein, I. S. Activity patterns in a cebus monkey group. Folia Primat., 1965, 3, 211-224.

Bernstein, I. S., \& Sharpe, L. G. Social roles in a rhesus monkey group. Behaviour, 1966, 26, 91-104.

Birch, H. G., \& Clark, G. Hormonal modification of social behavior: IV. The mechanism of estrogen induced dominance in chimpanzees. J. comp. physiol. Psychol., 1950, 43, 181-193.

Brady, J. V., Schreiner, L., Geller, I., \& Kling, A. Subcortical mechanisms in emotional behavior: The effect of rhinencephalic injury upon the acquisition and retention of a conditioned avoidance response in cats. J. comp. physiol. Psychol., 1954, 47, $179-186$.

Brody, E. B., \& Rosvold, H. E. Influence of prefontal lobotomy on social interaction in a monkey group. Psychosom. Med., 1952, 14, 406-415. 
Brown, T. S., Rosvold, H. E., \& Mishkin, M. Olfactory discrimination after temporal lobe lesions in monkeys. J. comp. physiel. Psychol., 1963, 56, 190-195.

Bunne11, B. N. Amygdaloid lesions and social dominance in the hooded rat. Paper read at Southern Soc. Phil. Psychol., New Orleans, April, 1966.

Bunnel1, B. N., Sodetz, F. J., \& Shalloway, D. M. The effects of septal and amygdaloid lesions on aggressiveness and social dominance in the hamster. Paper read at Psychon. Soc., Chicago, October, 1965.

Carpenter, C. R. A field study in Siam of the behavior and social relations of the gibbon. Comp. psychol. Monog., 1940, 16, 1-212.

Carpenter, C. R. Societies of monkeys and apes. Biol. Symposia. $1942, \underline{8}, 177-204$.

Delgado, J. M. R. Pharmacological modifications of social behavior. In W. D. M. Paton (Ed.), Pharmacological analysis of central nervous action. Vol. 8. Oxford: Pergamon Press, 1962, 265292 .

Delgado, J. M R. Cerebral heterostimulation in a monkey colony. Science, $1963,141,161-163$.

Delgado, J. M. R. Free behavior and brain stimulation. In C. C. Pfeiffer and J. R. Smythies (Eds.), International Review of Neurobiology. Vol. 8. New York: Academic Press, 1964, 349449 .

Delgado, J. M. R. Chronic radio-stimulation of the brain in monkey colonies. Excerpta Medica Int. Congr., 1965, 87, 365-371. (a)

Delgado, J. M. R. Pharmacology of spontaneous and conditioned behavior in the monkey. Proc. 2nd Int. Pharmacol. Congr., Prague. New York: Pergamon Press, 1965. (b)

Downer, J. L. deC. Interhemispheric Integration in the visual system. In V. B. Mountcastle (Ed.) Interhemispheric relations and cerebral dominance. Baltimore: Johns Hopkins Press, 1962, Pp. 87-100.

Drachman, D. A., \& Ommaya, A. H. Memory and the hippocampal complex. Arch. Neuro1., 1964, 10, 411-425.

Fuller, J. L., Rosvold, H. E., \& Pribram, K. H. The effect of affective and cognitive behavior in the dog of lesions of the pyriform-amygdala-hippocampal complex. J. comp. physiol. Psychol., 1957, 50, 89-96. 
Gloor, P. Amygdala. In J. Field (Ed.), Handbook of physiology. Vo1. 2. Neurophysiology. Washington, D. C.: American Physiological Society, 1960. Pp. 1395-1420.

Gloor, P., \& Feinde1, W. Affective behaviour and temporal lobe. In M. Monnier (Ed.), Physiologie und Pathophysiologie des vegetativen Nervensystems. Stuttgart: Hippokrates--Verlag, 1963. Pp. 685-716.

Goddard, G. V. Functions of the amygdala. Psychol. Bu11., 1964, $\underline{62}, 89-109$.

Gooda11, J. Feeding behavior of wild chimpanzees--a preliminary report. Symp. Zoo1. Soc. Lond., 1963, 10, 39-47.

Green, J. K., Clemente, C. D., \& DeGroot, J. Rhinencephalic lesions and behaviour in cats. J. comp. Neurol., 1957, 108, 505-545.

Hamilton, C. L. Comparison of two methods of dominance testing in the monkey. Psychol. Rep., 1960, 6, 247-250.

Hore1, J. A., Treichler, F. R., \& Meyer, D. Coercive behavior in the rhesus monkey. J. comp. physiol. Psychol., 1963, 56, 208210 .

Horvath, F. E. Effects of basolateral amygdalectomy on three types of avoidance behavior in cats. J. comp. physiol. Psychol., 1963 , $\underline{56}, 380-389$.

Kaufmann, J. H. Social relations of adult males in a free ranging band of rhesus monkeys. In S. A. Altmann (Ed.), Social communication among primates. Chicago: University of Chicago Press, 1966. In press.

King, F. A. Effect of septal and amygdaloid lesions on emotional behaviour and conditioned avoidance responses in the rat. J. nerv. ment. Dis., 1958, 126, 57-63.

King, F. A., \& Meyer, P. M. Effects of amygdaloid lesions upon septal hyperemotionality in the rat. Science, 1958, 128, 655-656.

Kling, A., \& Hutt, P. J. Effect of hypothalamic lesions on the amygdala syndrome in the cat. Arch. Neurol. Psychiat., 1958, 79, 511-517.

Kluver, H., \& Bucy, P. C. "Psychic blindness" and other symptoms following bilateral temporal lobectomy in rhesus monkeys. Am. J. Physiol., 1937, 119, 352-353.

Kluver, H., \& Bucy, P. C. Preliminary analysis of functions of the temporal lobes in monkeys. Arch. Neurol. Psychiat., 1939, 42, 979-1000. 
Kummer, H., \& Kurt, F. Social units of a free-living population of hamadryas baboons. Folia Primat., 1963, 1, 4-19.

Leary, R. W., \& Slye, D. Dominance reversal in drugged monkeys. J. Psychol., 1959, 48, 227-235.

Leary, K. W., \& Stynes, A. J. Tranquilizer effects in the social status, motivation and learning of monkeys. Arch. Gen. Psychiat., 1959, 1, 499-505.

MacLean, P. D. Mirror display in the squirrel monkey, Saimiri sciureus. Science, 1964, 146, 950-952.

MacLean, P. D., \& Delgado, J. M. R. Electrical and chemical stimulation of frontotemporal portion of limbic system in the waking animal. EEG clin. Neurophysiol., 1953, 5, 91-100.

Mahut, H., \& Cordeau, J. P. Spatial reversal deficit in monkeys with amygdalo-hippocampal ablations. Exp. Neurol., 1963, I,
426-434.

Mason, J. W. Plasma 17-hydroxycorticosteroid levels during electrical stimulation in the amygdaloid complex in conscious monkeys. Amer. J. Physiol., 1959, 196, 44-48.

Mason, W. A. The effects of social restriction on the behavior of rhesus monkeys: I. Free social behavior. J. comp. physiol. Psychol., 1960, 53, 582-589.

Mason, W. A. The effects of social restriction on the behavior of rhesus monkeys: III. Dominance tests. J. comp. physiol. Psychol., 1961, 54, 694-699.

Masserman, J. H., Levitt, M., McAvoy, T., Kling, A., \& Pechtel, C. The amygdalae and behaviour. Amer. J. Psychiat., 1958, 115,
14-17.

Miller, R. E., \& Banks, J. H., Jr. The determination of social dominance in monkeys by a competitive avoidance method. J. comp. physiol. Psychol., 1962, 55, 137-141.

Miller, R. E., Murphy, J. V., \& Mirsky, I. A. The modification of social dominance in a group of monkeys by interanimal conditioning. J. comp. physiol. Psychol., 1955, 48, 392-396.

Miller, R. E., \& Murphy, J. V. Social interaction of rhesus monkeys: I. Food-getting dominance as a dependent variable. J.Soc. Psychol., 1956, 44, 249-255.

Mirsky, A. F. The influence of sex hormones on social behavior in monkeys. J. comp. physiol. Psychol., 1955, 48, 327-335. 
Mirsky, A. F., Rosvold, H. E., \& Pribram, K. H. Effects of cingulectomy on social behavior in monkeys. J. Neurophysiol., $1957, \underline{20}, 588-601$.

Mishkin, M. Visual discrimination performance following partial ablations of the temporal lobe: II. Ventral surface vs. hippocampys. J. comp. physiol. Psychol., 1954, 47, 187-193.

Murphy, M. V., \& Miller, R. E. The manipulation of dominance in monkeys with conditioned fear. J. abnorm. soc. Psychol., $1956,53,244-248$.

Nauta, W. J. H. Fibre degeneration following lesions of the amygdaloid complex in the monkey. J. Anat., 1961, 95, 515-531.

Orbach, J., Milner, B., \& Rasmussen, T. Learning and retention in monkeys and amygdala-hippocampus resection. Arch. Neurol., $1960, \underline{3}, 230-251$.

Pinto-Hamuy, T., Santibanez, G., Gonzales, C., \& Vicencio, E. Changes in behavior and visual discrimination performances after selective ablations of the temporal cortex. J. comp. physio1. Psychol., 1957, 50, 379-385.

Ploog, D. W., Blitz, J., \& Ploog, F. Studies on social and sexual behavior of the squirrel monkey (Saimiri sciureus). Folia Primat., 1963, 1, 29-66.

Plotnik, R. J., King, F. A., \& Roberts, L. An objective analysis of social dominance in the squirrel monkey. Proc. Amer. Psychol. Assn., 1965, 1, 109-110.

Plotnik, R. J., King, F. A., \& Roberts, L. Comparison of social organization in two New World monkeys: Saimiri scuireus and Cebus albifrons. Proc. Amer. Psychol. Assn. In press.

Pribram, K. H., \& Mishkin, M. Simultaneous and successive visual discrimination by monkeys with inferotemporal lesions. J. comp. physiol. Psychol., 1955, 48, 198-202.

Pribram, K. H., \& Weiskrantz, L. A comparison of the effects of medial and lateral cerebral resection on conditioned avoidance in monkeys. J. comp. physiol. Psychol., 1957, 50, 74-80.

Reynolds, V. An outline of the behavior and social organization of forest-living chimpanzees. Folia Primat., 1963, 1, 95-102.

Robinson, E. The effect of amygdalectomy of fear-motivated behavior in rats. J. comp. physiol. Psychol., 1963, 56, 814-820.

Rosvold, H. E., Mirsky, A. F., \& Pribram, K. H. Influence of amygdalectomy on social behavior in monkeys. J. comp. physiol. Psychol., 1954, 47, 173-178. 
Sawa, M., \& Delgado, J. M. R. Amygdala unitary activity in the unrestrained cat. EEG clin. Neurophysiol., 1963, 15, 637650.

Schaller, G. The mountain gorilla: ecology and behavior. Chicago: University Chicago Press, 1963.

Schreiner, L., \& Kling, A. Behavioral changes following rhinencephalic injury in cats. J. Neurophysiol., 1953, 16, 643-659.

Schwartzbaum, J. S. Changes in reinforcing properties of stimuli following ablation of the amygdaloid complex in monkeys. J. comp. physiol. Psychol., 1960, 53, 388-396.

Schwartzbaum, J. S. Some characteristics of 'amygdaloid hyperphagia' in monkeys. Amer. J. Psychol., 1961, 74, 252-259.

Schwartzbaum, J. S. Visually reinforced behavior following ablation of the amygdaloid complex in monkeys. J. comp. physiol. Psychol., 1964, 57, 340-347.

Schwartzbaum, J. S. Discrimination behavior after amygdalectomy in monkeys: visual and somesthetic learning and perceptual capacity. J. comp. physiol. Psychol., 1965, 60, 314-319.

Schwartzbaum, J. S., \& Pribram, K. H. The effects of amygdalectomy in monkeys on transposition along a brightness continuum. J. comp. physiol. Psychol., 1960, 53, 396-399.

Schwartzbaum, J. S., Bowman, R. E., \& Holdstock, L. Visual exploration in the monkey following ablation of the amygdaloid complex. J. comp. physiol. Psychol., 1964, 57, 453-456.

Schwartzbaum, J. S., \& Poulos, D. A. Discrimination behavior after amygdalectomy in monkeys: learning set and discrimination reversals. J. comp. physiol. Psychol., 1965, 60, 320-328.

Southwick, C. H. Patterns of intergroup social behavior in primates, with special reference to rhesus and howling monkeys. Ann. N. Y. Acad. Sci., 1962, 102; 436-454.

Speigel, E. A., Miller, H. R., \& Oppenheimer, M. J. Forebrain and rage reactions. J. Neurophysiol., 1940, $\underline{3}$, 538-548.

Stepien, I. S., Cordeau, J. P., \& Rasmussen, T. The effect of temporal lobe and hippocampal lesions on auditory and visual recent memory in monkeys. Brain, 1960, 83, 470-489.

Terzian, H., \& Ore, G. D. Syndrome of Kluver and Bucy reproduced in man by bilateral removal of the temporal lobes. Neurology, $1955, \underline{5}, 373-380$. 
Walker, A. E., Thompson, A. F., \& McQueen, J. D. Behavior and the temporal rhinencephalon in the monkey. Johns Hopkins Bull., 1953, 93, 65-93.

Warren, J. M. Primate learning in comparative perspective. In A. M. Schrier, H. F. Harlow, \& F. Stollnitz (Eds.), Behavior of nonhuman primates. New York: Academic Press, 1965. Pp. 249-281.

Weiskrantz, L. Behavioral changes with ablation of the amygdaloid complex in monkeys. J. comp. physiol. Psychol., 1956, 49,
381-391.

Weiskrantz, L., \& Wilson, W. A., Jr. The effect of ventral rhinencephalic lesions on avoidance thresholds in monkeys. J. comp. physiol. Psychol., 1958, 51, 167-171.

Wendt, R., \& Albe-Fessard, D. Sensory responses of the amygdala with special reference to somatic afferent pathways. In Physiologie de l'hippocampe. Paris: Centre National de la Recherche Scientifique, 1962. Pp. 171-200.

Wood, C. D. Behavioral changes following discrete lesions of temporal lobe structures. Neurology, 1958, 8, 215-220.

Wood, J. W. Loss of aggressiveness in wild rats following lesions in the rhinencephalon. Int. Physiol. Congr. Abstr., 1956, 20, 978-979. 


\section{BIOGRAPHICAL SKETCH}

Rodney J. Plotnik was born April 15, 1939, in Browerville, Minnesota. He attended St. John's Preparatory School and was graduated in June, 1957. In June, 1961, the author received his B.A. degree from St. John's University with a major in Sociology and a minor in Psychology. He received his M.S. degree from the University of Miami in August, 1963, with a major in Psychology. In August, 1966, he received his Ph.D. degree from the University of Florida with a major in Psychology and a minor in Medical Sciences. His area of specialization is physiological psychology.

The author was appointed Research Assistant at the University of Miami from 1961 to 1963 and at the University of Florida from 1963 to 1964 . During $1965-1966$ he was a National Institute of Mental Health Research Fellow. The author is married to the former Michaelene Mylet of Denver, Colorado. 
This dissertation was prepared under the direction of the chairman of the candidate's supervisory committee and has been approved by all members of that committee. It was submitted to the Dean of the College of Arts and Sciences and to the Graduate Council, and was approved as partial fulfillment of the requirements for the degree of Doctor of Philosophy.

August 13, 1966

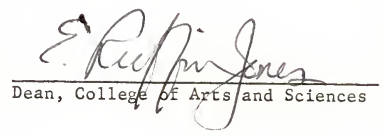

Dean, Graduate School

Supervisory Committee:

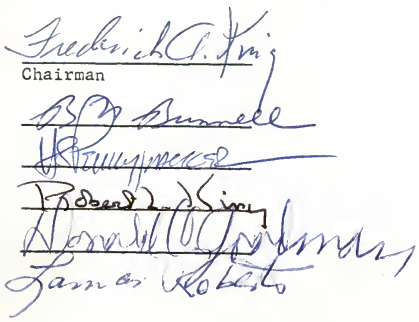

